\title{
Continuum random-phase approximation for gamma transition between excited states in neutron-rich nuclei
}

\author{
Teruyuki Saito \\ Graduate School of Science and Technology, \\ Niigata University, Niigata 950-2181, Japan \\ Masayuki Matsuo \\ Department of Physics, Faculty of Science. Niigata University, Niigata 950-2181, Japan \\ (Dated: May 17, 2021)
}




\begin{abstract}
A characteristic feature of collective and particle-hole excitations in neutron-rich nuclei is that many of them couple to unbound neutron in continuum single-particle orbits. The continuum random phase approximation (cRPA) is a powerful many-body method that describes such excitations, and it provides a scheme to evaluate transition strengths from the ground state. In an attempt to apply cRPA to the radiative neutron capture reaction, we formulate in the present study an extended scheme of cRPA that describes gamma-transitions from the excited states under consideration, which decay to low-lying excited states as well as the ground state. This is achieved by introducing a non-local one-body operator which causes transitions to a low-lying excited state, and describing a density-matrix response against this operator. As a demonstration of this new scheme, we perform numerical calculation for dipole, quadrupole, and octupole excitations in ${ }^{140} \mathrm{Sn}$, and discuss E1 and E2 transitions decaying to low-lying $2_{1,2}^{+}$and $3_{1}^{-}$states. The results point to cases where the branching ratio to the low-lying states is larger than or comparable with that to the ground state. We discuss key roles of collectivity and continuum orbits in both initial and final states.
\end{abstract}

\title{
I. INTRODUCTION
}

Theoretical and experimental studies of neutron-rich nuclei have been performed extensively in recent years, and they revealed peculiar features which are related to the small neutron separation energy or the weak binding of the last neutrons. Examples include the pygmy dipole resonance or the soft dipole excitation, which are considered as a new type of collective excitation or a continuum particle-hole excitations where a neutron is brought into unbound scattering state [1 4]. In the laboratory experiments, such exotic modes of excita- 
tion are observed in the excitation reactions such as the photo-absorption or the Coulomb or nuclear dissociation processes [5-7]. In the nature, neutron-rich nuclei play important role in the r-process nucleosynthesis, and it has been pointed out that the pygmy or the soft dipole excitations might influence the radiative neutron-capture reaction and resultant abundance of r-process nuclei [8, 9].

The radiative neutron-capture reaction are usually considered in terms of two different mechanisms: the statistical or compound nuclear $(\mathrm{CN})$ process and the direct capture $(\mathrm{DC})$ process [9]. The CN process is dominant in nuclei with relatively large neutron separation energy and high level density while the DC process becomes dominant in nuclei close to neutron-drip line [9 11]. For the CN process, the Hauser-Feshbach statistical model is assumed and the role of the exotic excitation modes are usually taken into account via the gamma-decay strength function [9]. For the DC process, however, the exotic modes need to be described explicitly as a doorway state of the neutron capture, and also do gammadecays from the populated excited state. Such theoretical descriptions of the DC process, applicable to the medium and heavy neutron-rich nuclei (relevant to the r-process), have not been formulated, in our knowledge, except our preceding study [12. The DC models applied so far to medium and heavy nuclei adopt the independent particle model [13 18], in which the collective effect is neglected. The semi-direct model[19, 20] takes into account the effect of the giant resonance is proposed, but it is essentially the same as the independent particle model as far as the r-process neutron-capture at very low neutron energy is concerned.

In the previous publication [12], we adopted the continuum quasiparticle random-phase approximation (cQRPA) based on the density functional theory to describe the DC process via the exotic excitation modes. We describe the Coulomb excitation or photo-absorption of an even-even neutron-rich nucleus $A$, leading to an excited state $\mathrm{A}^{*} \rightarrow(\mathrm{A}-1)+\mathrm{n}$ which 
may emit a neutron if the excitation energy exceeds the neutron threshold. Collective correlations are taken into account in the linear response framework to calculate the strength function, and the Green's function method [21 23] enables us to include the unbound single-neutron state with a scattering wave. By decomposing the strength function into different channels of $(\mathrm{A}-1)+\mathrm{n}$ with a method of Zangwill and Soven [24] and using the reciprocity theorem, we obtain the cross section of the radiative direct neutron-capture $(\mathrm{A}-1)+\mathrm{n} \rightarrow \mathrm{A}^{*} \rightarrow \mathrm{A}+\gamma$. We remark however that further improvement is needed in this approach since the gamma transitions $\mathrm{A}^{*} \rightarrow \mathrm{A}^{* *}+\gamma$ decaying to low-lying excited states $A^{* *}$ also occur in reality.

In the present study and in subsequent papers, we intend to extend the approach of Ref. [12] in order to describe the radiative direct neutron capture process $(\mathrm{A}-1)+\mathrm{n} \rightarrow \mathrm{A}^{*} \rightarrow \mathrm{A}^{* *}+\gamma$ taking place via collective and non-collective states $\mathrm{A}^{*}$ decaying to low-lying excited states $\mathrm{A}^{* *}$ of the synthesized nucleus. We shall proceed in two steps. As the first step, given in the present publication, we formulate a new method to calculate the transition matrix elements of the gamma transitions between two excited states $A^{*}$ and $A^{* *}$. Calculation of the transition matrix elements between excited states are straightforward if both states are discrete bound states and their wave functions are explicitly given on discrete basis. A novel feature of the formulation proposed here is that we use the linear response theory which is able to describe continuum excited state $\mathrm{A}^{*}$ located above the neutron separation energy. This is an essential requirement in applying to the neutron-rich nuclei near the drip-line. The second step, an application to the radiative direct neutron-capture reaction will be given in a forthcoming paper.

In section 2, we formulate a linear response theory extended to calculate transition matrix elements between excited states. For this purpose we define a non-local one-body operator 
introduced to evaluate the matrix elements. Applying the linear response formalism to this non-local operator, we obtain a new type of strength function which describes excitation modes in the continuum and transition matrix elements with respect to a low-lying excited state. This extended linear response formalism enables us to evaluate the branching ratios for gamma-decays from the continuum excited states to different low-lying excited states as well as the ground states. In section 3, we demonstrate applicability of the present approach by performing a numerical calculation for a neutron-rich nucleus ${ }^{140} \mathrm{Sn}$, and discuss the dipole, quadrupole and octupole excitations including the continuum particle-hole modes and the giant resonances, and the transitions from/to low-lying $2^{+}$and $3^{-}$states. We draw conclusions in section 5 .

\section{THEORY}

In the first three subsections we introduce the extended formalism of the continuum random-phase approximation which describes the transitions between RPA excited states. We then provide, in the following subsections, a detailed formulation for an application to a spherical nucleus with a $j$-shell closed configuration.

\section{A. Strength function for transitions between RPA excited states}

We shall describe excited states $\{|k\rangle\}$ by means of the random phase approximation (RPA) to oscillations around the ground state $|0\rangle$. The standard RPA formalism provides a scheme to calculate the transition matrix elements $\langle k|\hat{M}| 0\rangle$ and the strength function $S(\hat{M} ; \hbar \omega) \equiv \sum_{k}|\langle k|\hat{M}| 0\rangle|^{2} \delta\left(\hbar \omega-\left(E_{k}-E_{0}\right)\right)$ for a one-body operator $\hat{M}$, e.g., an electromagnetic multipole operator[25]. 
In the present paper, we consider another RPA excited state $|i\rangle$, for instance, the low-lying collective state with a character of surface vibration, and we intend to describe the transition matrix elements $\langle k|\hat{M}| i\rangle$ between the low-lying state $|i\rangle$ and the RPA excited states $\{|k\rangle\}$ under consideration. Since we consider neutron-rich (or proton-rich) nuclei and the situation where the RPA excited states $\{|k\rangle\}$ are populated via the direct neutron (proton) capture reaction, we shall treat the RPA excited states $\{|k\rangle\}$ as those embedded in the continuum spectrum above the neutron (proton) separation energy. It is then appropriate to describe $\{|k\rangle\}$ by means of the continuum RPA, i.e., the linear response theory using the Green's function technique[21, 22].

We introduce a strength function for transitions between RPA excited states $|i\rangle$ and $\{|k\rangle\}$ :

$$
S(\hat{M} ; i ; \Delta E)=\sum_{k}|\langle k|\hat{M}| i\rangle|^{2} \delta\left(\Delta E-\left(E_{k}-E_{i}\right)\right) .
$$

Here $|i\rangle$ is fixed and $|k\rangle$ runs over all excited states described by the continuum RPA. The RPA excited states are generally described in terms of the mode creation operator $\hat{O}^{\dagger}$, a linear combination of the particle-hole and hole-particle excitations, which is written as

$$
\hat{O}_{i}^{\dagger}=\sum_{p h}\left\{X_{p h}^{i} a_{p}^{\dagger} a_{h}-Y_{p h}^{i} a_{h}^{\dagger} a_{p}\right\}
$$

e.g. for the state $|i\rangle=\hat{O}_{i}^{\dagger}|0\rangle$. Using the mode creation operator, the strength function (1) can be rewritten as

$$
\begin{aligned}
S(\hat{M} ; i ; \hbar \omega) & \equiv \sum_{k}|\langle k|\hat{M}| i\rangle|^{2} \delta\left(\hbar \omega-\left(E_{k}-E_{0}\right)\right) \\
& =\sum_{k}\left|\left\langle k\left|\left[\hat{M}, \hat{O}_{i}^{\dagger}\right]\right| 0\right\rangle\right|^{2} \delta\left(\hbar \omega-\left(E_{k}-E_{0}\right)\right)=S(\hat{F} ; \hbar \omega) .
\end{aligned}
$$

Note that the second expression can be regarded as a strength function for transitions from the ground state $|0\rangle$ caused by a newly defined operator $\hat{F} \equiv\left[\hat{M}, O_{i}^{\dagger}\right]$. The replacement by the commutator is valid under the quasi-boson approximation [25] $\left[\hat{O}_{i}^{\dagger}, \hat{O}_{k}\right]=\delta_{i k}$, which 
is equivalent to keeping the leading orders of the boson expansion of $\hat{M}, \hat{O}$, and $\hat{O}^{\dagger}$. For simplicity the excitation energy $\hbar \omega=E_{k}-E_{0}$ is used in Eq.(3) in place of the transition energy $\Delta E=E_{k}-E_{i}$ in Eq.(1).

We remark here that the commutator $\hat{F}=\left[\hat{M}, O_{i}^{\dagger}\right]$ is a one-body but non-local operator. In fact, for the multipole moment

$$
\hat{M}=\int d x f\left(\mathbf{r}_{x}\right) \hat{\rho}(x), \quad f\left(\mathbf{r}_{x}\right) \equiv r_{x}^{L} Y_{L M}\left(\Omega_{x}\right),
$$

the operator $\hat{F}$ is

$$
\begin{gathered}
\hat{F}=\iint d x d y F(x, y) \psi^{\dagger}(x) \psi(y) \\
F(x, y) \equiv\left(f\left(\mathbf{r}_{x}\right)-f\left(\mathbf{r}_{y}\right)\right) \sum_{p h}\left\{X_{p h}^{i} \phi_{p}(x) \phi_{h}^{*}(y)-Y_{p h}^{i} \phi_{h}(x) \phi_{p}^{*}(y)\right\} .
\end{gathered}
$$

Here $\psi^{\dagger}(x), \psi(x)$ and $\hat{\rho}(x)=\psi^{\dagger}(x) \psi(x)$ are the creation, annihilation and density operators of nucleon with a shorthand notation of the coordinate and the spin variables $x \equiv\left(\mathbf{r}_{x}, \sigma_{x}\right)$ while $\phi_{p}(x)$ and $\phi_{h}(x)$ are single-particle wave functions of the particle and hole orbits, respectively. The isospin variable is omitted for simplicity. The integral $\int d x$ represents $\int d x \equiv \sum_{\sigma_{x}} \int d \mathbf{r}_{x}$

The expression (3) allows us to formulate the linear response of the system against an external perturbation provided by the non-local one-body operator $\hat{F}=\left[\hat{M}, O_{i}^{\dagger}\right]$. A new feature is that we need to consider a response of the non-local density, i.e. the density matrix $\rho(x, y)=\langle\hat{\rho}(x, y)\rangle$ with $\hat{\rho}(x, y)=\psi^{\dagger}(y) \psi(x)$. The response in the frequency domain is given by

$$
\delta \rho(x, y, \omega)=\iint d x^{\prime} d y^{\prime} R\left(x, y ; y^{\prime}, x^{\prime} ; \omega\right) F\left(x^{\prime}, y^{\prime}\right)
$$

with a response function generalized to the density matrix, which is formally expressed as

$$
R\left(x, y ; y^{\prime}, x^{\prime} ; \omega\right) \equiv \sum_{k}\left\{\frac{\langle 0|\hat{\rho}(x, y)| k\rangle\left\langle k\left|\hat{\rho}\left(y^{\prime}, x^{\prime}\right)\right| 0\right\rangle}{\hbar \omega-\hbar \omega_{k}+i \eta}-\frac{\left\langle 0\left|\hat{\rho}\left(y^{\prime}, x^{\prime}\right)\right| k\right\rangle\langle k|\hat{\rho}(x, y)| 0\rangle}{\hbar \omega+\hbar \omega_{k}+i \eta}\right\}
$$


Here $\eta$ is a positive infinitesimal constant and $\hbar \omega_{k}=E_{k}-E_{0}$ is the excitation energy of the RPA excited states $\{|k\rangle\}$. The strength function $S(\hat{F} ; \hbar \omega)$ in Eq. 3 is given by

$$
S(\hat{F} ; \hbar \omega)=-\frac{1}{\pi} \operatorname{Im} \iint d x d y F^{*}(x, y) \delta \rho(x, y, \omega)
$$

using the density-matrix response $\delta \rho(x, y, \omega)$.

Note that the strength function can be expressed also as

$$
S(\hat{F} ; \hbar \omega)=-\frac{1}{\pi} \operatorname{Im} \int d x f^{*}(x)\left\{\int d y \bar{\rho}_{i}^{(\operatorname{tr}) *}(x, y) \delta \rho(x, y, \omega)-\int d y \bar{\rho}_{i}^{(\operatorname{tr}) *}(y, x) \delta \rho(y, x, \omega)\right\}
$$

obtained by inserting Eq. (6) into Eq. (9). Here we introduced a quantity

$$
\bar{\rho}_{i}^{(\operatorname{tr})}(x, y) \equiv \sum_{p h}\left\{X_{p h}^{i} \phi_{p}(x) \phi_{h}^{*}(y)-Y_{p h}^{i} \phi_{h}(x) \phi_{p}^{*}(y)\right\}
$$

to represent the second factor in Eq.(6) associated with the RPA state $|i\rangle$. We call it the pseudo transition density-matrix of $|i\rangle$ since it has the same structure as the transition density-matrix

$$
\rho_{i}^{(\operatorname{tr})}(x, y) \equiv\langle 0|\hat{\rho}(x, y)| i\rangle=\left\langle 0\left|\left[\hat{\rho}(x, y), \hat{O}_{i}^{\dagger}\right]\right| 0\right\rangle=\sum_{p h}\left\{X_{p h}^{i} \phi_{p}(x) \phi_{h}^{*}(y)+Y_{p h}^{i} \phi_{h}(x) \phi_{p}^{*}(y)\right\}
$$

except the sign of the second term related to the backward amplitudes $Y_{p h}^{i}$.

\section{B. Extended linear response equation}

Since the operator $\hat{F}$ is a one-body, though non-local, operator, it is possible to formulate the linear response on the basis of the time-dependent Kohn-Sham theory, or the timedependent Hartree-Fock theory. Separating the time-dependent selfconsistent field $U[\rho]=$ 
$U_{0}+U_{\text {ind }}$ into the stationary part $U_{0}$ associated with the ground state and the induced field $U_{\text {ind }}=\left(\frac{\delta U}{\delta \rho}\right) \delta \rho$, the density-matrix response is given by

$$
\delta \rho(x, y, \omega)=\iint d x^{\prime} d y^{\prime} R_{0}\left(x, y ; y^{\prime}, x^{\prime} ; \omega\right)\left(v_{i n d}\left(x^{\prime}, y^{\prime}, \omega\right)+F\left(x^{\prime}, y^{\prime}\right)\right)
$$

in terms of the unperturbed response function

$$
R_{0}\left(x, y ; y^{\prime}, x^{\prime} ; \omega\right) \equiv \sum_{p h}\left\{\frac{\langle 0|\hat{\rho}(x, y)| p h\rangle\left\langle p h\left|\hat{\rho}\left(y^{\prime}, x^{\prime}\right)\right| 0\right\rangle}{\hbar \omega-\left(\epsilon_{p}-\epsilon_{h}\right)+i \eta}-\frac{\left\langle 0\left|\hat{\rho}\left(y^{\prime}, x^{\prime}\right)\right| p h\right\rangle\langle p h|\hat{\rho}(x, y)| 0\rangle}{\hbar \omega+\left(\epsilon_{p}-\epsilon_{h}\right)+i \eta}\right\}
$$

for uncorrelated particle-hole states $|p h\rangle=a_{p}^{\dagger} a_{h}|0\rangle$. The unperturbed response function is also given as

$$
\begin{array}{r}
R_{0}\left(x, y ; y^{\prime}, x^{\prime} ; \omega\right)=\sum_{\epsilon_{i}<\epsilon_{f}}\left\{\phi_{i}^{*}(y) G_{0}\left(x, x^{\prime}, \epsilon_{i}+\hbar \omega+i \eta\right) \phi_{i}\left(y^{\prime}\right)\right. \\
\left.+\phi_{i}^{*}\left(x^{\prime}\right) G_{0}\left(y^{\prime}, y, \epsilon_{i}-\hbar \omega-i \eta\right) \phi_{i}(x)\right\},
\end{array}
$$

using the single-particle Green's function $G_{0}\left(x, x^{\prime}, e\right)=\left\langle x\left|\left(e-\hat{h}_{0}\right)^{-1}\right| x^{\prime}\right\rangle=\sum_{i} \phi_{i}^{*}(x) \phi_{i}\left(x^{\prime}\right)(e-$ $\left.\epsilon_{i}\right)^{-1}$ for the mean-field Hamiltonian $\hat{h}_{0}=\hat{t}+U_{0}$. The Green's function allows one to describe the single-particle states in the continuum and hence RPA excited states embedded in the continuum spectrum above the particle separation energy.

In the following, we assume that the induced field is local and spin-independent. $v_{\text {ind }}\left(x^{\prime}, y^{\prime}, \omega\right)=v_{i n d}\left(\mathbf{r}_{x^{\prime}}, \omega\right) \delta\left(x^{\prime}-y^{\prime}\right)=\frac{\delta U}{\delta \rho}\left(\mathbf{r}_{x^{\prime}}, \omega\right) \delta \rho\left(\mathbf{r}_{x^{\prime}}\right) \delta\left(\mathbf{r}_{x^{\prime}}-\mathbf{r}_{y^{\prime}}\right) \delta_{\sigma_{x^{\prime}} \sigma_{y^{\prime}}}$. In this case, the density-matrix response $\delta \rho(y, x, \omega)$ is given by

$\delta \rho(x, y, \omega)=\int d x^{\prime} R_{0}\left(x, y ; x^{\prime}, x^{\prime} ; \omega\right) \frac{\delta U}{\delta \rho}\left(\mathbf{r}_{x^{\prime}}\right) \delta \rho\left(\mathbf{r}_{x^{\prime}}, \omega\right)+\iint d x^{\prime} d y^{\prime} R_{0}\left(x, y ; y^{\prime}, x^{\prime} ; \omega\right) F\left(x^{\prime}, y^{\prime}\right)$.

For the local density response $\delta \rho\left(\mathbf{r}_{x}, \omega\right)=\sum_{\sigma_{x}} \delta \rho(x, y=x, \omega)$ appearing in the right hand 
side of Eq.16), we have an integral equation

$\delta \rho\left(\mathbf{r}_{x}, \omega\right)=\sum_{\sigma_{x}} \int d x^{\prime} R_{0}\left(x, x ; x^{\prime}, x^{\prime} ; \omega\right) \frac{\delta U}{\delta \rho}\left(\mathbf{r}_{x^{\prime}}\right) \delta \rho\left(\mathbf{r}_{x^{\prime}}, \omega\right)+\sum_{\sigma_{x}} \iint d x^{\prime} d y^{\prime} R_{0}\left(x, x ; y^{\prime}, x^{\prime} ; \omega\right) F\left(x^{\prime}, y^{\prime}\right)$

We can solve numerically the integral equation (17) by treating it as a linear equation on the mesh points in the coordinate space. Inserting the density response $\delta \rho\left(\mathbf{r}_{x}, \omega\right)$ into Eq. 16), the density-matrix response $\delta \rho(x, y, \omega)$ is obtained, and finally we can calculate the strength function $S(\hat{F} ; \hbar \omega)$ using Eq. 10. Note that the pseudo transition density-matrix

$\bar{\rho}_{i}^{(\operatorname{tr})}(x, y)$ of the state $|i\rangle$ can be calculated also within the linear response formalism (the continuum RPA formalism) as we discuss in Appendix A. Consequently all the calculations are done within the consistent framework of the continuum RPA.

\section{Transition densities and diagrammatic interpretation}

We first note that the transition densities for transitions between the ground state and the RPA excited states are calculated as

$$
\rho_{k}^{(\operatorname{tr})}(x) \equiv\langle 0|\hat{\rho}(x)| k\rangle=C \operatorname{Im} \delta \rho\left(x, \omega_{k}\right)
$$

for the local transition density, and similarly

$$
\rho_{k}^{(\operatorname{tr})}(x, y) \equiv\left\langle 0\left|\psi^{\dagger}(y) \psi(x)\right| k\right\rangle=C \operatorname{Im} \delta \rho\left(x, y, \omega_{k}\right)
$$

for the transition density-matrix. The density responses $\delta \rho\left(x, \omega_{k}\right)$ and $\delta \rho\left(x, y, \omega_{k}\right)$ are solutions of Eqs.16) and (17) at the excitation energy $E_{k}-E_{0}=\hbar \omega_{k}$ of the state $|k\rangle$. Here $C$ is a normalization constant which is determined to reproduce the transition strength evaluated from the strength function. 
Now we shall consider the transition density for the transition between the RPA excited states, i.e. the one between $|i\rangle$ and $|k\rangle$ :

$$
\rho_{i, k}^{(\operatorname{tr})}(x) \equiv\langle i|\hat{\rho}(x)| k\rangle=\left\langle 0\left|\left[\hat{O}_{i}, \hat{\rho}(x)\right]\right| k\right\rangle
$$

Using the commutation relation

$$
\left[\hat{O}_{i}, \hat{\rho}(x)\right]=\int d y\left\{\bar{\rho}_{i}^{(\operatorname{tr}) *}(x, y) \psi^{\dagger}(y) \psi(x)-\bar{\rho}_{i}^{(\operatorname{tr}) *}(y, x) \psi^{\dagger}(x) \psi(y)\right\}
$$

the transition density is given as

$$
\rho_{i, k}^{(\operatorname{tr})}(x)=\int d y\left\{\bar{\rho}_{i}^{(\operatorname{tr}) *}(x, y) \rho_{k}^{(\operatorname{tr})}(x, y)-\bar{\rho}_{i}^{(\operatorname{tr}) *}(y, x) \rho_{k}^{(\operatorname{tr})}(y, x)\right\}
$$

expressed as a convolution of the transition density-matrix $\rho_{k}^{(\operatorname{tr})}(x, y)$ for the state $|k\rangle$ and the pseudo transition density-matrix $\bar{\rho}_{i}^{(\operatorname{tr})}(x, y)$ for the state $|i\rangle$.

The transition density is expressed also in terms of the forward and backward amplitudes of the mode creation operators:

$$
\begin{aligned}
\rho_{i, k}^{(\operatorname{tr})}(x)= & \sum_{p p^{\prime} h} X_{p h}^{i *} X_{p^{\prime} h}^{k} \phi_{p}^{*}(x) \phi_{p^{\prime}}(x)-\sum_{p h h^{\prime}} X_{p h}^{i *} X_{p h^{\prime}}^{k} \phi_{h}(x) \phi_{h^{\prime}}^{*}(x) \\
& +\sum_{p p^{\prime} h} Y_{p h}^{i *} Y_{p^{\prime} h}^{k} \phi_{p}^{*}(x) \phi_{p^{\prime}}(x)-\sum_{p h h^{\prime}} Y_{p h}^{i *} Y_{p h^{\prime}}^{k} \phi_{h}(x) \phi_{h^{\prime}}^{*}(x) .
\end{aligned}
$$

It is possible to interpret each term using a diagrammatic representation as shown in Fig,1. Figure 1 (a) and (b), corresponding to the first and second terms of Eq. 23), represent actions of the operator on particle and hole components of the RPA states, respectively whereas Fig. 1 (c) and (d) are counterparts, the third and fourth terms, associated with the backward amplitudes of the RPA states.

The transition matrix elements $\langle i|\hat{M}| k\rangle=\int d x f(x) \rho_{i, k}^{(\operatorname{tr})}(x)$ between the RPA excited states for a one-body operator $\hat{M}$ is also represented in terms of the same diagrams. 


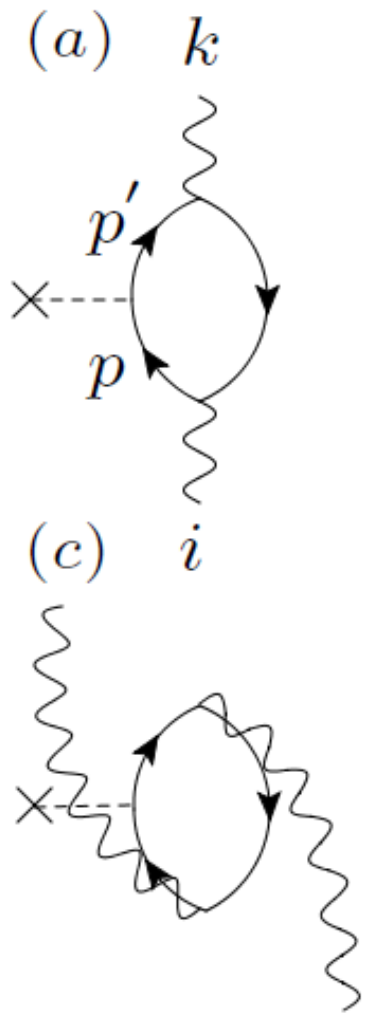

$(b)$

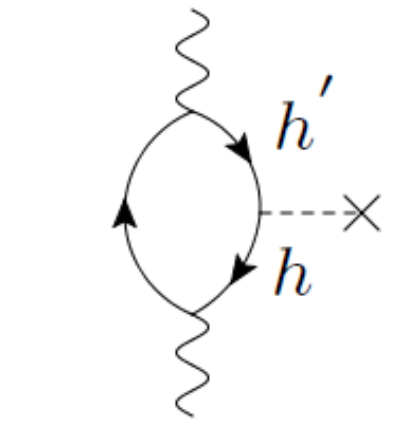

$(d)$

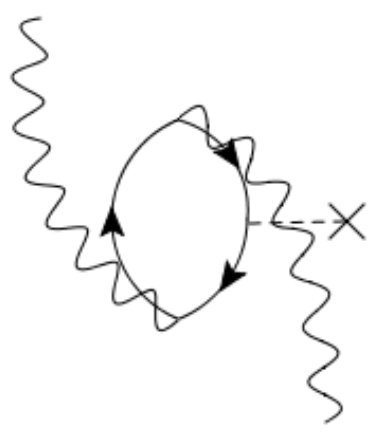

FIG. 1. The diagram representation of the transition density or the matrix element of a one-body operator between RPA excited states $|i\rangle$ and $|k\rangle$. See Eq.23 and the text.

\section{Spherical system}

In this section, we give explicit formulae which can be used in actual numerical calculation. Here the spherical symmetry of the ground state and the associated mean-field is assumed.

Suppose that the ground state $|0\rangle$, the RPA excited states $|k\rangle,|i\rangle$ and the transition operator $\hat{M}$ have the angular momentum quantum numbers $0^{+}, L M, L_{i} M_{i}$ and $\lambda \mu$, respectively. The operator $\hat{F}=\left[\hat{M}, \hat{O}_{i}^{\dagger}\right]$ is given the explicit rank $L M$ :

$$
\hat{F}_{L M} \equiv \sum_{\mu M_{i}}\left\langle\lambda \mu L_{i} M_{i} \mid L M\right\rangle\left[\hat{M}_{\lambda \mu}, \hat{O}_{i L_{i} M_{i}}^{\dagger}\right]
$$

Using this operator we evaluate the strength function for the transitions from the ground 
state $\left|0_{\mathrm{g}}^{+}\right\rangle$to excited RPA states $|k L M\rangle$

$$
\begin{aligned}
S\left(\hat{F}_{L} ; \mathrm{g}, L ; \hbar \omega\right) & \equiv \sum_{k M}\left|\left\langle k L M\left|\hat{F}_{L M}\right| 0_{\mathrm{g}}^{+}\right\rangle\right|^{2} \delta\left(\hbar \omega-\left(E_{k}-E_{0}\right)\right) \\
& =\sum_{k}\left|\left\langle k L|| \hat{F}_{L}|| 0_{\mathrm{g}}^{+}\right\rangle\right|^{2} \delta\left(\hbar \omega-\left(E_{k}-E_{0}\right)\right) .
\end{aligned}
$$

It is identical to the strength function

$$
S\left(\hat{M}_{\lambda} ; i L_{i}, L ; \hbar \omega\right) \equiv \sum_{k}\left|\left\langle k L|| \hat{M}_{\lambda}|| i L_{i}\right\rangle\right|^{2} \delta\left(\hbar \omega-\left(E_{k}-E_{0}\right)\right)=S\left(\hat{F}_{L} ; \mathrm{g}, L ; \hbar \omega\right)
$$

which describes reduced matrix elements for transitions from the RPA excited state $\left|i L_{i} M_{i}\right\rangle$ to a set of RPA excited states $\{|k L M\rangle\}$. Note that the angular quantum numbers $L_{i}$ and $L$ of the excited states are explicitly indicated to label the strength functions $(25)$ and $(26)$.

The density response and the density-matrix response caused by $\hat{F}_{L M}$ also have quantum numbers $L M$. These functions and the matrix element of $\hat{F}_{L M}$ are expanded by the spherical harmonics and the spin spherical harmonics as

$$
\begin{gathered}
\delta \rho\left(\mathbf{r}_{x}, \omega\right)=Y_{L M}\left(\hat{\mathbf{r}}_{x}\right) \frac{1}{r_{x}^{2}} \delta \rho_{L}\left(r_{x}, \omega\right), \\
\delta \rho(x, y, \omega)=\sum_{l j m, l^{\prime} j^{\prime} m^{\prime}} Y_{l^{\prime} j^{\prime} m^{\prime}}(\hat{x}) \frac{1}{\sqrt{2 j^{\prime}+1}}\left\langle j m L M \mid j^{\prime} m^{\prime}\right\rangle \frac{\delta \rho_{L, l^{\prime} j^{\prime}, l j}\left(r_{x}, r_{y}, \omega\right)}{r_{x} r_{y}} Y_{l j m}^{*}(\hat{y}), \\
F_{L M}(x, y)=\sum_{l j m, l^{\prime} j^{\prime} m^{\prime}} Y_{l^{\prime} j^{\prime} m^{\prime}}(\hat{x}) \frac{1}{\sqrt{2 j^{\prime}+1}}\left\langle j m L M \mid j^{\prime} m^{\prime}\right\rangle \frac{F_{L, l^{\prime} j^{\prime}, l j}\left(r_{x}, r_{y}\right)}{r_{x} r_{y}} Y_{l j m}^{*}(\hat{y}) .
\end{gathered}
$$

The extended linear response equations for the radial functions of the density responses are given as

$$
\begin{array}{r}
\delta \rho_{L, l^{\prime} j^{\prime}, l j}\left(r_{x}, r_{y}, \omega\right)=\left\langle l^{\prime} j^{\prime}|| Y_{L}|| l j\right\rangle \int d r_{x^{\prime}} R_{0, l^{\prime} j^{\prime}, l j}\left(r_{x}, r_{y} ; r_{x^{\prime}}, r_{x^{\prime}} ; \omega\right) \frac{\delta U}{\delta \rho}\left(r_{x^{\prime}}\right) \frac{1}{r_{x^{\prime}}^{2}} \delta \rho_{L}\left(r_{x^{\prime}}, \omega\right) \\
+\iint d r_{x^{\prime}} d r_{y^{\prime}} R_{0, l^{\prime} j^{\prime}, l j}\left(r_{x}, r_{y} ; r_{y^{\prime}}, r_{x^{\prime}} ; \omega\right) F_{L, l^{\prime} j^{\prime}, l j}\left(r_{x^{\prime}}, r_{y^{\prime}}\right),
\end{array}
$$




$$
\begin{array}{r}
\delta \rho_{L}\left(r_{x}, \omega\right)=\sum_{l j, l^{\prime} j^{\prime}}\left\{\frac{\left|\left\langle l^{\prime} j^{\prime}|| Y_{L}|| l j\right\rangle\right|^{2}}{2 L+1} \int d r_{x^{\prime}} R_{0, l^{\prime} j^{\prime}, l j}\left(r_{x}, r_{x} ; r_{x^{\prime}}, r_{x^{\prime}} ; \omega\right) \frac{\delta U}{\delta \rho}\left(r_{x^{\prime}}\right) \frac{1}{r_{x^{\prime}}^{2}} \delta \rho_{L}\left(r_{x^{\prime}}, \omega\right)\right. \\
\left.+\frac{\left\langle l^{\prime} j^{\prime}|| Y_{L}|| l j\right\rangle^{*}}{2 L+1} \iint d r_{x^{\prime}} d r_{y^{\prime}} R_{0, l^{\prime} j^{\prime}, l j}\left(r_{x}, r_{x} ; r_{y^{\prime}}, r_{x^{\prime}} ; \omega\right) F_{L, l^{\prime} j^{\prime}, l j}\left(r_{x^{\prime}}, r_{y^{\prime}}\right)\right\} .
\end{array}
$$

The explicit form of radial unperturbed response function $R_{0, l^{\prime} j^{\prime}, l j}$ is given in Appendix A and can be calculated using the exact single-particle Green's function. Note also that there holds a relation

$$
\delta \rho_{L}\left(r_{x}, \omega\right)=\frac{1}{2 L+1} \sum_{l j, l^{\prime} j^{\prime}}\left\langle l^{\prime} j^{\prime}|| Y_{L}|| l j\right\rangle^{*} \delta \rho_{L, l^{\prime} j^{\prime}, l j}\left(r_{x}, r_{x}, \omega\right) .
$$

The strength function $S\left(\hat{F}_{L} ; \mathrm{g}, L ; \hbar \omega\right)$ is given by

$$
\begin{aligned}
& S\left(\hat{F}_{L} ; \mathrm{g}, L ; \hbar \omega\right)=-\frac{1}{\pi} \operatorname{Im} \iint d r_{x} d r_{y} \sum_{\substack{l j \\
l^{\prime} j^{\prime}}} F_{L, l^{\prime} j^{\prime}, l j}^{*}\left(r_{x}, r_{y}\right) \delta \rho_{L, l^{\prime} j^{\prime}, l j}\left(r_{x}, r_{y}, \omega\right) \\
& =-\frac{1}{\pi} \operatorname{Im} \int d r_{x} f_{\lambda}^{*}\left(r_{x}\right) \\
& \times \sum_{l j, l^{\prime} j^{\prime}, l_{2} j_{2}}\left[\sqrt{2 L+1}(-)^{L+1}(-)^{j-j^{\prime}}\left\{\begin{array}{ccc}
\lambda & L_{i} & L \\
j & j^{\prime} & j_{2}
\end{array}\right\}\left\langle l^{\prime} j^{\prime}|| Y_{\lambda}|| l_{2} j_{2}\right\rangle^{*}\right. \\
& \times \int d r_{y} \bar{\rho}_{i L_{i}, l_{2} j_{2}, l j}^{\mathrm{tr}) *}\left(r_{x}, r_{y}\right) \delta \rho_{L, l^{\prime} j^{\prime}, l j}\left(r_{x}, r_{y}, \omega\right) \\
& +\sqrt{2 L+1}(-)^{L_{i}-\lambda}(-)^{j^{\prime}-j}\left\{\begin{array}{ccc}
\lambda & L_{i} & L \\
j^{\prime} & j & j_{2}
\end{array}\right\}\left\langle l_{2} j_{2}|| Y_{\lambda}|| l j\right\rangle^{*} \\
& \left.\times \int d r_{y} \bar{\rho}_{i L_{i}, l^{\prime} j^{\prime}, l_{2} j_{2}}^{\mathrm{tr}) *}\left(r_{y}, r_{x}\right) \delta \rho_{L, l^{\prime} j^{\prime}, l j}\left(r_{y}, r_{x}, \omega\right)\right] .
\end{aligned}
$$


Here is used the expression for the matrix element of the operator $\hat{F}_{L M}$

$$
\begin{array}{r}
F_{L, l^{\prime} j^{\prime}, l j}\left(r_{x}, r_{y}\right)=\sum_{l_{2} j_{2}} \sqrt{2 L+1}\left[(-)^{L+1}(-)^{j-j^{\prime}}\left\{\begin{array}{ccc}
\lambda & L_{i} & L \\
j & j^{\prime} & j_{2}
\end{array}\right\}\left\langle l^{\prime} j^{\prime}|| Y_{\lambda}|| l_{2} j_{2}\right\rangle f_{\lambda}\left(r_{x}\right) \bar{\rho}_{i L_{i}, l_{2} j_{2}, l j}^{(\operatorname{tr})}\left(r_{x}, r_{y}\right)\right. \\
\left.+(-)^{L_{i}-\lambda}(-)^{j^{\prime}-j}\left\{\begin{array}{ccc}
\lambda & L_{i} & L \\
j^{\prime} & j & j_{2}
\end{array}\right\}\left\langle l_{2} j_{2}|| Y_{\lambda}|| l j\right\rangle f_{\lambda}\left(r_{y}\right) \bar{\rho}_{i L_{i}, l^{\prime} j^{\prime}, l_{2} j_{2}}^{(\operatorname{tr})}\left(r_{x}, r_{y}\right)\right],
\end{array}
$$

with the pseudo radial transition density-matrix $\bar{\rho}_{i L_{i}, l^{\prime} j^{\prime}, l j}^{(\operatorname{tr})}$ for the low-lying discrete state $\left|i L_{i} M_{i}\right\rangle$

\section{E. Photoemission decays to low-lying states}

One can apply the above formulation to describe photoemission transitions from excited states in the continuum to a low-lying excited state. We consider a transition of multipole $\lambda$ from the excited state $|k L M\rangle$ in the continuum at energy $E_{k}$ to the low-lying bound excited state $\left|i L_{i} M_{i}\right\rangle$ at $E_{i}$. The transition probability[25], proportional to the reduced matrix element $B\left(M_{\lambda}, k L \rightarrow i L_{i}\right)=\frac{1}{2 L+1}\left|\left\langle i L_{i}|| \hat{M}_{\lambda}|| k L\right\rangle\right|^{2} \Delta E$, is given by

$$
T_{k L \rightarrow i L_{i}}=\frac{8 \pi(\lambda+1)}{\hbar \lambda((2 \lambda+1) ! !)^{2}}\left(\frac{E_{\gamma}}{\hbar c}\right)^{2 \lambda+1} \frac{1}{2 L+1} S\left(\hat{M}_{\lambda} ; i L_{i}, L ; E_{k}-E_{0}\right) \Delta E
$$

with $E_{\gamma}=E_{k}-E_{i}$ using the strength function $S\left(\hat{F}_{L} ; \mathrm{g}, L ; E_{k}-E_{0}\right)=S\left(\hat{M}_{\lambda} ; i L_{i}, L ; E_{k}-E_{0}\right)$. The energy interval $\Delta E$ is chosen arbitrarily small for the continuum $|k\rangle$ whereas in the case of discrete $|k\rangle$ it should be treated as an integral $\int_{E_{k}-\Delta E / 2}^{E_{k}+\Delta E / 2} S\left(\hat{F}_{L} ; \mathrm{g}, L ; E-E_{0}\right) d E$ to cover the associated peak structure of the strength function. 


\section{NUMERICAL EXAMPLE}

\section{A. Setting}

We shall describe electromagnetic transitions in neutron-rich nucleus ${ }^{140} \mathrm{Sn}$ in order to demonstrate the present theory. The neutron separation energy in this nucleus is predicted as small as $S_{n} \sim 3 \mathrm{MeV}$ by the Hartree-Fock calculations [26], and it may be one of the isotopes which play roles in the r-process neutron-capture. We notice also that the pair correlation of neutrons is expected to be weak due to a single- $j$ closed configuration.

We focus on excited states with spin-parity $1^{-}, 2^{+}$and $3^{-}$where characteristic excited states are expected to emerge both in low-lying and high-lying regions. Examples are the soft dipole mode and the giant dipole resonance for $1^{-}$, the low-lying quadrupole state and the isoscalar/isovector giant quadrupole resonances for $2^{+}$, and the low-lying octupole collective states for $3^{-}$as well as continuum particle-hole excitations above the neutron separation energy. We shall discuss electric multipole transitions ( E1, E2 and E3) which occur among these states and the ground state.

The numerical calculations is performed with the following setting. We use a WoodsSaxon potential in place of the Hartree-Fock mean-field $U_{0}$ and a Skyrme-type contact interaction as the residual two-body force $v_{p h}=\delta U / \delta \rho$, given by

$$
v_{p h}\left(\mathbf{r}, \mathbf{r}^{\prime}\right)=\left\{t_{0}\left(1+x_{0} P_{\sigma}\right)+\frac{t_{3}}{12}\left(1+x_{3} P_{\sigma}\right) \rho(r)\right\} \delta\left(\mathbf{r}-\mathbf{r}^{\prime}\right)
$$

where we adopt the same parameter as Ref.[22]: $t_{0}=f \times(-1100) \mathrm{fm}^{3} \mathrm{MeV}, t_{3}=f \times$ $16000 \mathrm{fm}^{6} \mathrm{MeV}, x_{0}=0.5, x_{3}=1, P_{\sigma}$ is the spin-exchange operator. The Woods-Saxon parameter is that of Ref.[22], and the Coulomb potential for a uniform charge sphere is included for protons. Since the Woods-Saxon potential is not the self-consistent potential derived from the interaction, we impose an approximate self-consistency condition on this 
residual interaction by multiplying a renormalization factor $f=0.749$ to $v_{p h}$ so that the spurious mode of the center of mass motion, appearing as a RPA eigen mode with multipole $1^{-}$, has zero excitation energy.

We obtain single-particle wave function and the single-particle Green's function $G_{0}$ by solving the radial Schroödinger equation with the Runge-Kutta method up to a maximal radius $R_{\max }=20 \mathrm{fm}$ (with interval $\Delta r=0.2 \mathrm{fm}$ ). At $r=R_{\max }$ the single-particle wave function is connected to the asymptotic wave, i.e, the Hankel function with an appropriate (complex) wave number. All the single-particle partial waves are included, i.e., up to the maximum orbital angular momentum $l_{\max }=l_{h, \max }+\max \left\{L_{i}, L\right\}+1$ where $l_{h, \max }$ is the largest among the hole orbits. The small imaginary constant $\eta$ in the response equation is set to $\eta=0.1 \mathrm{MeV}$ in most cases although it is chosen much smaller in specific cases.

Figure 2 shows the strength functions for the transitions from the ground state to the excited states with spin-parity $L^{\pi}=1^{-}, 2^{+}$and $3^{-}$: (a) the E1 strength function $S\left(D_{\mathrm{IV}} ; \mathrm{g}, 1^{-} ; E\right)$ for $1^{-}$states, excited by $\hat{D}_{\mathrm{IV}}=\frac{N}{A} \sum_{i, \text { proton }}\left(r Y_{1 \mu}\right)_{i}-\frac{Z}{A} \sum_{i, \text { neutron }}\left(r Y_{1 \mu}\right)_{i}$ (b) the E2 and isoscalar quadrupole strength functions $S\left(Q_{\mathrm{p}} ; \mathrm{g}, 2^{+} ; E\right)$ and $S\left(Q_{\mathrm{IS}} ; \mathrm{g}, 2^{+} ; E\right)$ for $2^{+}$states with $\hat{Q}_{\mathrm{p}}=\sum_{i, \text { proton }}\left(r^{2} Y_{2 \mu}\right)_{i}$ and $\hat{Q}_{\mathrm{IS}}=\hat{Q}_{\mathrm{p}}+\hat{Q}_{\mathrm{n}}$, (c) the E3 and isoscalar octupole strength functions for $3^{-}$states with $\hat{O}_{\mathrm{p}}=\sum_{i \text {,proton }}\left(r^{3} Y_{3 \mu}\right)_{i}$ and $\hat{O}_{\mathrm{IS}}=\hat{O}_{\mathrm{p}}+\hat{O}_{\mathrm{n}}$.

Table II shows the single-particle orbits. The energy of the neutron $2 f_{7 / 2}$ orbit (the Fermi energy ) is $e_{2 f_{7 / 2}}=-2.59 \mathrm{MeV}$. As seen in Fig 2(a), there exist low-energy dipole strength which is discributed continuously above the neutron threshold $S_{n}=2.59 \mathrm{MeV}$. This continuum strength is brought mainly by the neutron particle-hole excitation from the last occupied $2 f_{7 / 2}$ orbit to the continuum $d_{5 / 2}$ orbit. (We denote this configuration as " $\nu\left[\left(\text { cont. } d_{5 / 2}\right)\left(2 f_{7 / 2}\right)^{-1}\right]_{1^{-}} "$ in the following. $)$ The large strength distributed around $E \approx$ 11-15 MeV corresponds to the giant dipole resonance (GDR). From the strength functions of 
$2^{+}$states, we focus on the lowest two discrete states at $E_{2_{1}^{+}}=0.888 \mathrm{MeV}, E_{2_{2}^{+}}=1.093 \mathrm{MeV}$ lying below the neutron threshold. The strength distributions around $E \approx 12-13 \mathrm{MeV}$ and $E \approx 21-25 \mathrm{MeV}$ are the isoscalar giant quadrupole resonance (ISGQR) and the isovector one (IVGQR). Two peaks around $E \approx 5$ and $\approx 6 \mathrm{MeV}$ consists mainly of proton particlehole excitations $\pi\left[\left(1 g_{7 / 2}\right)\left(1 g_{9 / 2}\right)^{-1}\right]_{2^{+}}$and $\pi\left[\left(2 d_{5 / 2}\right)\left(1 g_{9 / 2}\right)^{-1}\right]_{2^{+}}$while the enhanced isoscalar strengths of these peaks indicate contributions of neutron particle-hole components. For $3^{-}$ states, we notice a low-lying discrete state at $E_{3_{1}^{-}}=1.768 \mathrm{MeV}$, which has a character of the octupole surface vibration.

In the following discussion we pick up the three discrete states, the first and second $2^{+}$ states and the first $3^{-}$states as the low-lying excited state $\left|i L_{i}\right\rangle$. We shall describe the matrix element $\left\langle i L_{i}|| \hat{M}_{\lambda}|| k L\right\rangle$ of multipole transitions between these low-lying states and the RPA excited states $|k L\rangle$ lying above the neutron threshold in the $1^{-}, 2^{+}$and $3^{-}$sectors.

We describe the E1, E2 and E3 transitions using the operators $\hat{M}_{\lambda}=\hat{D}_{\mathrm{IV}}, \hat{Q}_{\mathrm{p}}$ and $\hat{O}_{\mathrm{p}}$ with the bare charge of nucleons.

The present theory takees into account the collectivity/correlation in both the initial and final states on top of the continuum effects. We shall demonstrate this feature by comparing two calculations in which the correlation/collectivity of the excited states either included or neglected. We drop off the induced field $v_{\text {ind }}=(\delta U / \delta \rho) \delta \rho$ in the linear response equations when we neglect the correlation. In this case the excited states become unperturbed particlehole excitations.

\section{B. $1^{-}$states: soft dipole excitation and GDRs}

Let us first consider excited dipole states and discuss E1 and E2 transitions from the low-lying $2_{1,2}^{+}$and $3_{1}^{-}$states. 


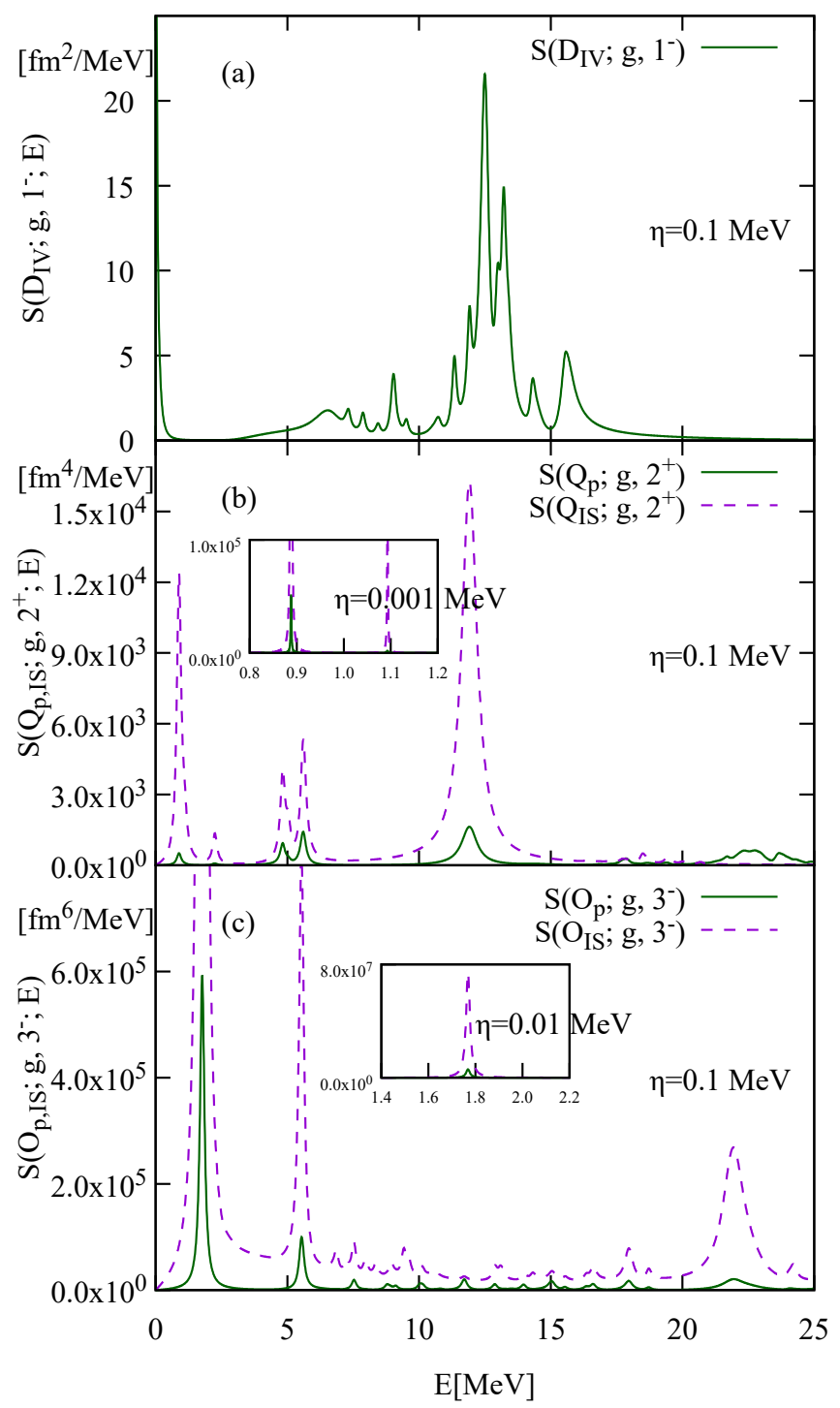

FIG. 2. (a)The E1 strength function $S\left(D_{\mathrm{IV}} ; \mathrm{g}, 1^{-} ; E\right)$ for excited $1^{-}$states in ${ }^{140} \mathrm{Sn}$, calculated with $\eta=0.1 \mathrm{MeV}$. The horizontal axis is the excitation energy of the $1^{-}$states. (b)The E2 and isoscalar quadrupole strength functions $S\left(Q_{\mathrm{p}} ; \mathrm{g}, 2^{+} ; E\right)$ and $S\left(Q_{\mathrm{IS}} ; \mathrm{g}, 2^{+} ; E\right)$ for excited $2^{+}$ states, plotted with green solid and magenta dashed curves, respectively. The inset shows the result with $\eta=0.001 \mathrm{MeV}$, in which the $2_{1}^{+}$and $2_{2}^{+}$states are separately seen at excitation energy $E_{2_{1}^{+}}=0.888 \mathrm{MeV}$ and $E_{2_{2}^{+}}=1.093 \mathrm{MeV}$. (c) The E3 and isoscalar octupole strength functions $S\left(O_{\mathrm{p}} ; \mathrm{g}, 3^{-} ; E\right)$ and $S\left(O_{\mathrm{IS}} ; \mathrm{g}, 3^{-} ; E\right)$ for excited $3^{-}$states. The lowest energy peak is the $3_{1}^{-}$state with $E_{3_{1}^{-}}=1.768 \mathrm{MeV}$. 
TABLE I. Single-particle energies of the adopted Woods-Saxon potential for ${ }^{140} \mathrm{Sn}$. Several orbits around the Fermi energy (indicated by lines) are listed.

\begin{tabular}{ccccc}
\hline neutron $\epsilon[\mathrm{MeV}]$ & & \multicolumn{2}{c}{ proton $\epsilon[\mathrm{MeV}]$} \\
\hline $2 f_{5 / 2}$ & -0.31 & & $1 h_{11 / 2}$ & -11.40 \\
$3 p_{1 / 2}$ & -0.81 & & $2 d_{3 / 2}$ & -11.61 \\
$3 p_{3 / 2}$ & -1.46 & & $2 d_{5 / 2}$ & -14.06 \\
$1 h_{9 / 2}$ & -1.53 & & $1 g_{7 / 2}$ & -15.08 \\
$2 f_{7 / 2}$ & -2.59 & & $1 g_{9 / 2}$ & -19.97 \\
$1 h_{11 / 2}$ & -6.64 & & $2 p_{1 / 2}$ & -21.75 \\
$3 s_{1 / 2}$ & -8.65 & & $2 p_{3 / 2}$ & -23.02 \\
$2 d_{3 / 2}$ & -8.65 & & $1 f_{5 / 2}$ & -24.81 \\
$2 d_{5 / 2}$ & -10.40 & & & \\
$1 g_{7 / 2}$ & -10.96 & & & \\
$1 g_{9 / 2}$ & -14.64 & & & \\
\hline
\end{tabular}

1. $\quad$ E1 transition $2_{1,2}^{+} \rightarrow 1^{-}$

Figure 3 (a) shows the strength function $S\left(D_{\mathrm{IV}} ; 2_{1,2}^{+}, 1^{-} ; E\right)$ for the E1 transitions from the low-lying $2_{1,2}^{+}$states to excited $1^{-}$states. The strength function $S\left(D_{\mathrm{IV}} ; \mathrm{g}, 1^{-} ; E\right)$ for the E1 transitions from the ground state is also plotted in the lower panel for comparison. It is seen that the strength distribution for the transitions from $2_{1}^{+}$and $2_{2}^{+}$is very different from that from the ground state. We note here that the strength function $S\left(D_{\mathrm{IV}} ; 2_{1,2}^{+}, 1^{-} ; E\right)$ has little strength in the GDR region $(E \approx 12-17 \mathrm{MeV})$ while there exists a rather sharp peak 
around $E \approx 9 \mathrm{MeV}$. We note also continuous distribution of the strength for the $1^{-}$states in the region of the soft dipole excitation $S_{1 n}=2.59<E \lesssim 7 \mathrm{MeV}$. However the shape of this continuum strength is different from that in the E1 strength function $S\left(D_{\mathrm{IV}} ; \mathrm{g}, 1^{-} ; E\right)$ for the transition from the ground state.

The above behaviors can be explained with the following picture. We first note that the correlation in the low-lying $2_{1,2}^{+}$states is rather simple; their main structures are mixtures of lowest-energy neutron particle-hole excitations $\nu\left[\left(1 h_{9 / 2}\right)\left(2 f_{7 / 2}\right)^{-1}\right]_{2^{+}}$and $\nu\left[\left(3 p_{3 / 2}\right)\left(2 f_{7 / 2}\right)^{-1}\right]_{2^{+}}$ (with excitation energies $E=1.06 \mathrm{MeV}$ and $E=1.13 \mathrm{MeV}$, respectively) as indicated by the forward amplitudes shown in Table II. (Note that other particle-hole configurations have small amplitudes $\left|X_{p h}\right|<0.1$. ) Given this feature, main components which contribute to the E1 transitions between $2_{1,2}^{+}$and $1^{-}$particle-hole excitatioins are rather limited, as is listed in Fig, 4 . Figure 5 shows unpertrubed E1 transitions associated with these components, i.e. transitions from the neutron particle-hole states $\nu\left[\left(1 h_{9 / 2}\right)\left(2 f_{7 / 2}\right)^{-1}\right]_{2^{+}}$and $\nu\left[\left(3 p_{3 / 2}\right)\left(2 f_{7 / 2}\right)^{-1}\right]_{2^{+}}$to uncorrelated $1^{-}$particle-hole states. From comparison of the strength functions from $2_{1,2}^{+}$(Fig. 3 (a)) and the unperturbed strength from $\nu\left[\left(3 p_{3 / 2}\right)\left(2 f_{7 / 2}\right)^{-1}\right]_{2^{+}}$(Fig. 5), we find that the continuum strength in the soft dipole region $S_{1 n}=2.59<E \lesssim 7 \mathrm{MeV}$ originates from the component $\nu\left[\left(3 p_{3 / 2}\right)\left(2 f_{7 / 2}\right)^{-1}\right]_{2^{+}} \rightarrow$ $\nu\left[\left(\text { cont. } d_{5 / 2}\right)\left(2 f_{7 / 2}\right)^{-1}\right]_{1^{-}}$in which the E1 operator causes a single-particle transition of a neutron in the $3 p_{3 / 2}$ orbit to the continuum $d_{5 / 2}$ orbit. Absolute strengths are well explained by the mixing amplitude of $\nu\left[\left(3 p_{3 / 2}\right)\left(2 f_{7 / 2}\right)^{-1}\right]_{2^{+}}$, and it reflects the uncorrelated particle-hole nature of the soft dipole states. The narrow peak around $E \approx 9 \mathrm{MeV}$ is due to $\nu\left[\left(3 p_{3 / 2}\right)\left(2 f_{7 / 2}\right)^{-1}\right]_{2^{+}} \rightarrow \nu\left[\left(3 p_{3 / 2}\right)\left(2 d_{5 / 2}\right)^{-1}\right]_{1^{-}}$with E1 transition of a neutron hole $\left(2 f_{7 / 2}\right)^{-1} \rightarrow\left(2 d_{5 / 2}\right)^{-1}$. In this case, however, the strengths of this peak deviates from simple estimation based on the the mixing amplitudes. This is probably because the $1^{-}$states 


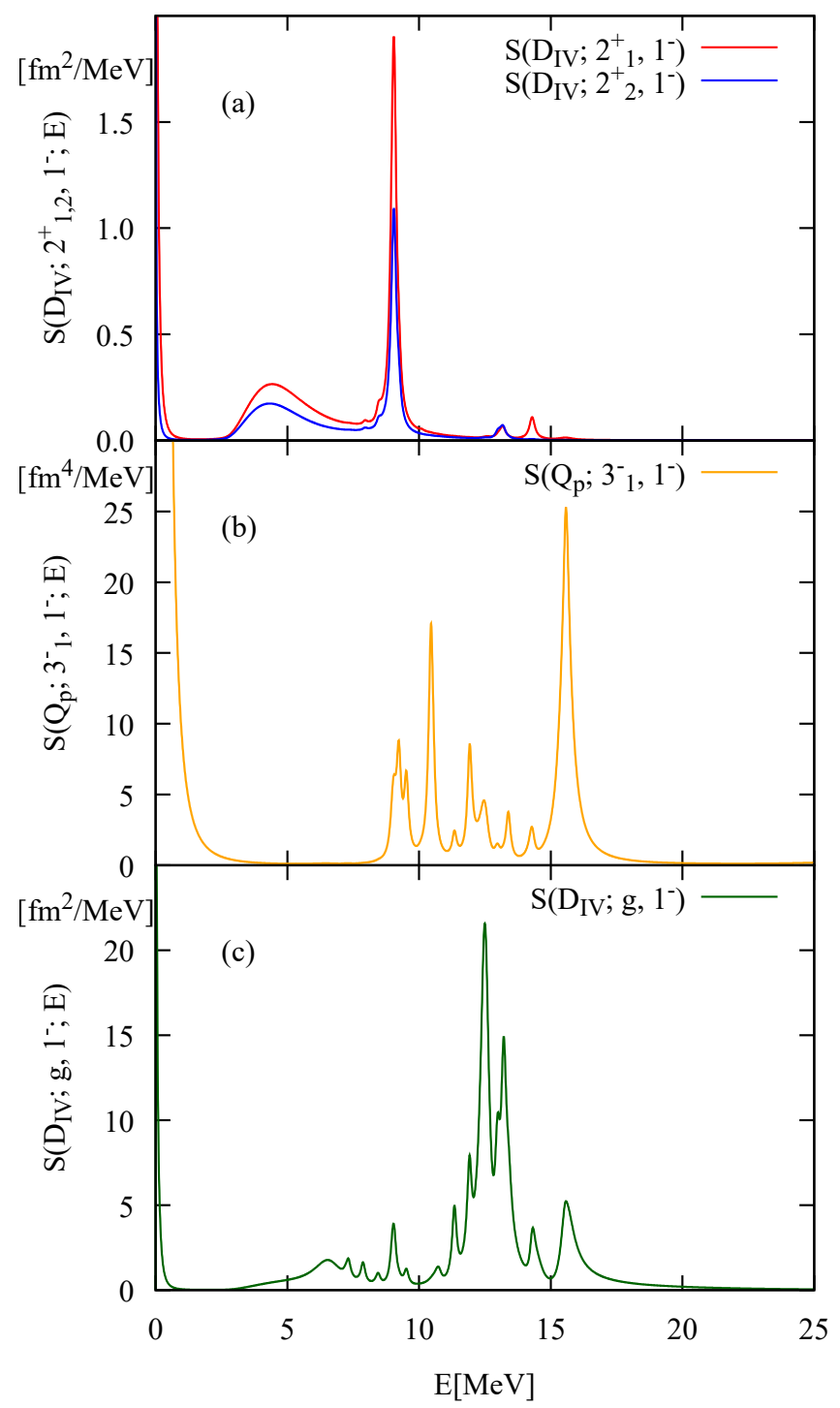

FIG. 3. (a)The E1 strength functions $S\left(D_{\mathrm{IV}} ; 2_{1,2}^{+}, 1^{-} ; E\right)$ for transitions from $2_{1,2}^{+}$to $1^{-}$states. (b) The E2 strength function $S\left(Q_{\mathrm{p}} ; 3_{1}^{-}, 1^{-} ; E\right)$ for transitions from $3_{1}^{-}$to $1^{-}$states. (c) The E1 strength function $S\left(D_{\mathrm{IV}} ; \mathrm{g}, 1^{-} ; E\right)$ for transitions from the ground state to $1^{-}$states. The horizontal axis is the excitation energy of the $1^{-}$states.

in this energy region is not simple particle-hole excitations. Small peaks around $E \approx 13$ $\mathrm{MeV}$ can be attributed to a contribution of $\nu\left[\left(1 h_{9 / 2}\right)\left(2 f_{7 / 2}\right)^{-1}\right]_{2^{+}} \rightarrow \nu\left[\left(1 h_{9 / 2}\right)\left(1 g_{9 / 2}\right)^{-1}\right]_{1^{-}}$ (Fig, $4(\mathrm{c}))$. The lack of the strength in the GDR region and higher is a consequence of the 
TABLE II. The RPA forward amplitudes $X_{p h}$ of the $2_{1}^{+}$and $2_{1}^{+}$states. Particle-hole configurations with large amplitude $\left|X_{p h}\right|>0.1$ are listed. The RPA backward and forward amplitudes $X_{p h}$ and $Y_{p h}$ are calculated using a method of Ref. [27]

\begin{tabular}{lcc}
\hline neutron config. & $X_{p h}^{2_{1}^{+}}$ & $X_{p h}^{2_{2}^{+}}$ \\
\hline$\left(1 h_{9 / 2}\right)\left(2 f_{7 / 2}\right)^{-1}$ & -0.601 & 0.791 \\
$\left(3 p_{3 / 2}\right)\left(2 f_{7 / 2}\right)^{-1}$ & 0.789 & 0.600 \\
\hline
\end{tabular}
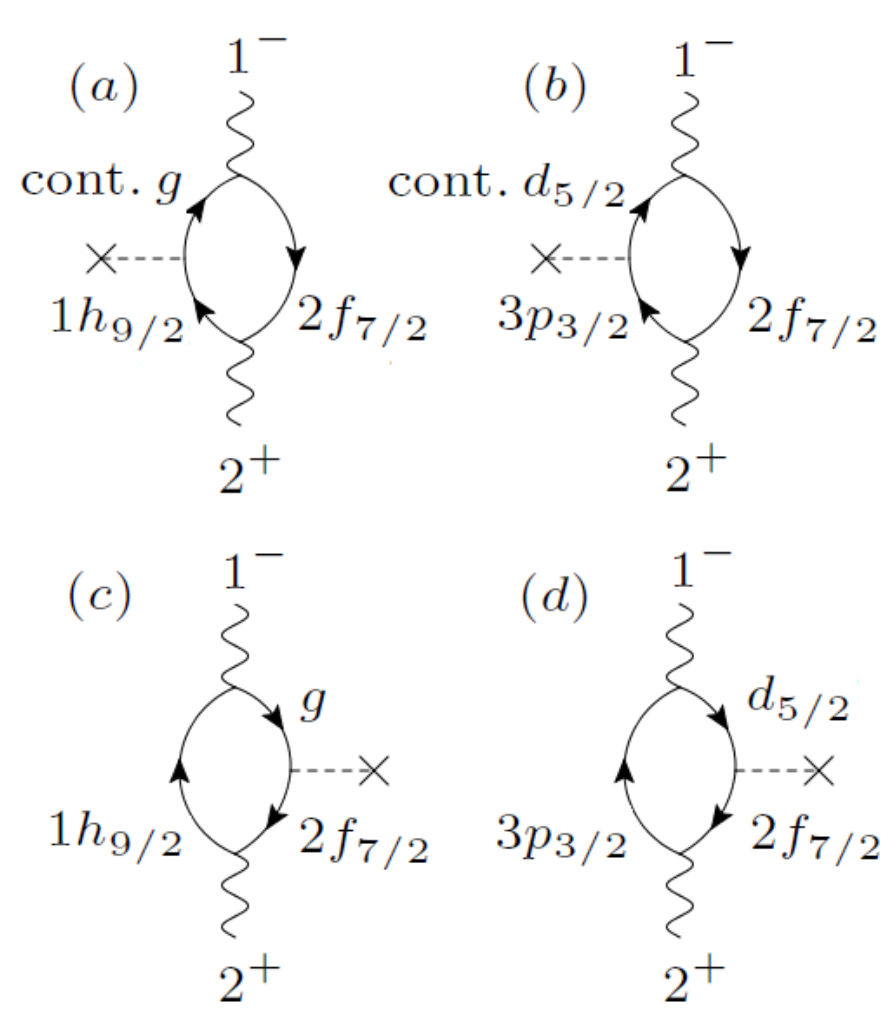

FIG. 4. The diagrams representing dominant components of transition between the exited $1^{-}$ states and the low-lying $2_{1,2}^{+}$in ${ }^{140} \mathrm{Sn}$.

small number of dominant particle-hole configurations in the low-lying $2_{1,2}^{+}$states. 


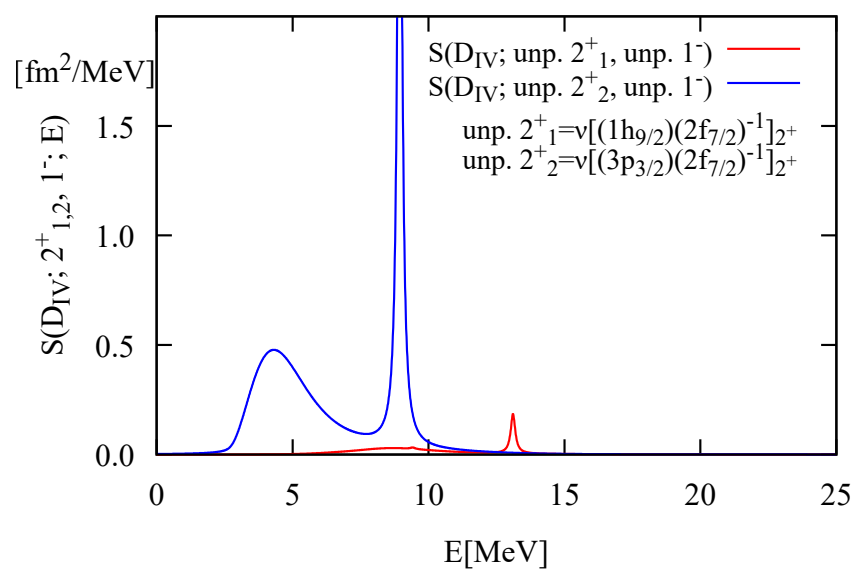

FIG. 5. The E1 strength functions $S\left(D_{\mathrm{IV} ;} \nu\left[\left(1 h_{9 / 2}\right)\left(2 f_{7 / 2}\right)^{-1}\right]_{2^{+}}, 1^{-} ; E\right)$ and $S\left(D_{\mathrm{IV}} ; \nu\left[\left(3 p_{3 / 2}\right)\left(2 f_{7 / 2}\right)^{-1}\right]_{2^{+}}, 1^{-} ; E\right) \quad$ for transitions from the neutron $1 \mathrm{p}-1 \mathrm{~h}$ states $\nu\left[\left(1 h_{9 / 2}\right)\left(2 f_{7 / 2}\right)^{-1}\right]_{2^{+}}$and $\nu\left[\left(3 p_{3 / 2}\right)\left(2 f_{7 / 2}\right)^{-1}\right]_{2^{+}}$to unperturbed $1^{-}$states. The horizontal axis is the excitation energy of the $1^{-}$states. The strength function $S\left(D_{\mathrm{IV}} ; \nu\left[\left(3 p_{3 / 2}\right)\left(2 f_{7 / 2}\right)^{-1}\right]_{2^{+}}, 1^{-} ; E\right)$ has a peak at $E=8.94 \mathrm{MeV}$ with the maximum value $3.67 \mathrm{fm}^{2} / \mathrm{MeV}$.

\section{E2 transition $3_{1}^{-} \rightarrow 1^{-}$}

The E2 transitions from the low-lying $3_{1}^{-}$state to the dipole states, shown in Fig. $3(\mathrm{~b})$, exhibits behavior different from that of Fig. $3(\mathrm{a})$. The low-lying $3_{1}^{-}$state has the collective character of the surface octupole vibration, including many particle-hole configurations of not only neutrons but also protons, as seen in the RPA amplitudes (Table III). Here relevant to the E2 transition are proton particle-hole configurations in $3_{1}^{-}$since we use the bare charge for the E2 operator. It is seen in Fig. 3(b) that there is no visible strength in the region of the soft dipole transition $\left(S_{1 n}<E \lesssim 7 \mathrm{MeV}\right)$, and this is due to the neutron character of the soft dipole excitation. It is seen also that there exists several peaks in the energy region $8 \lesssim E \lesssim 17 \mathrm{MeV}$, in contrast to the E1 transitions from the $2_{1,2}^{+}$. This originates from the relatively large number of proton particle-hole configurations mixed in the collective $3_{1}^{-}$ 
TABLE III. The RPA forward amplitudes $X_{p h}$ of the $3_{1}^{-}$state. Particle-hole configurations with large amplitude $\left|X_{p h}\right|>0.1$ are listed. The neutron single-particle orbit $1 i_{13 / 2}$ is a resonance in the continuum.

\begin{tabular}{cccc}
\hline neutron config. & $X_{p h}^{3_{1}^{-}}$ & proton config. & $X_{p h}^{3_{1}^{-}}$ \\
\hline$\left(1 i_{13 / 2}\right)\left(2 f_{7 / 2}\right)^{-1}$ & 0.831 & $\left(1 h_{11 / 2}\right)\left(1 g_{9 / 2}\right)^{-1}$ & -0.285 \\
$\left(1 i_{13 / 2}\right)\left(1 h_{11 / 2}\right)^{-1}$ & 0.354 & $\left(1 g_{7 / 2}\right)\left(2 p_{1 / 2}\right)^{-1}$ & 0.203 \\
$\left(1 h_{9 / 2}\right)\left(2 d_{3 / 2}\right)^{-1}$ & -0.299 & $\left(2 d_{5 / 2}\right)\left(2 p_{1 / 2}\right)^{-1}$ & 0.176 \\
$\left(1 h_{9 / 2}\right)\left(1 g_{7 / 2}\right)^{-1}$ & -0.189 & $\left(2 d_{5 / 2}\right)\left(2 p_{3 / 2}\right)^{-1}$ & 0.135 \\
$\left(2 f_{5 / 2}\right)\left(3 s_{1 / 2}\right)^{-1}$ & 0.134 & $(1 j 15 / 2)\left(1 g_{9 / 2}\right)^{-1}$ & 0.129 \\
$\left(2 g_{9 / 2}\right)\left(2 f_{7 / 2}\right)^{-1}$ & 0.133 & $\left(2 f_{7 / 2}\right)\left(1 g_{9 / 2}\right)^{-1}$ & -0.129 \\
$\left(2 f_{5 / 2}\right)\left(2 d_{3 / 2}\right)^{-1}$ & -0.126 & $\left(2 d_{3 / 2}\right)\left(2 p_{3 / 2}\right)^{-1}$ & -0.120 \\
$\left(3 p_{3 / 2}\right)\left(2 d_{3 / 2}\right)^{-1}$ & -0.112 & $\left(3 p_{3 / 2}\right)\left(1 g_{9 / 2}\right)^{-1}$ & -0.103 \\
$(2 j 15 / 2)\left(1 g_{9 / 2}\right)^{-1}$ & 0.108 & $\left(1 g_{7 / 2}\right)\left(1 f_{5 / 2}\right)^{-1}$ & 0.102 \\
$\left(2 f_{5 / 2}\right)\left(1 g_{7 / 2}\right)^{-1}$ & -0.101 & & \\
\hline
\end{tabular}

state.

\section{Decay branching ratio from $1^{-}$states}

Combining the above results, we shall discuss the branching ratio for the photo-emission decays from excited $1^{-}$states to the ground state, $2_{1,2}^{+}$and $3_{1}^{-}$states. The result is shown in Fig.6(a). It is seen that the soft dipole states in the energy region $S_{1 n}<E \lesssim 7 \mathrm{MeV}$ decays not only to the ground state but also to both $2_{1}^{+}$and $2_{2}^{+}$states with sizable branching ratio $20-40 \%$ (summing $2_{1}^{+}$and $2_{2}^{+}$). This reflects that the neutron single-particle transition 


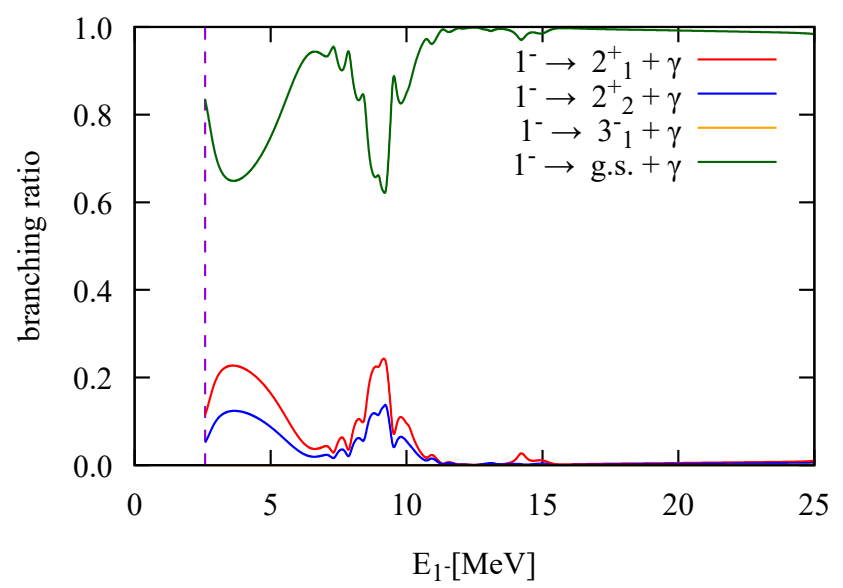

FIG. 6. The branching ratios of photo-emission decays from the excited $1^{-}$states to the ground state (green curve), the $2_{1}^{+}$state (red curve) and the $2_{2}^{+}$state (blue curve) in ${ }^{140} \mathrm{Sn}$. Horizontal axis is the excitation energy of the $1^{-}$states. The neutron separation energy $S_{1 n}=2.59 \mathrm{MeV}$ is indicated by dotted line. The branching ratio to the $3_{1}^{-}$state is invisibly small.

$\left(\right.$ cont. $\left.d_{5 / 2}\right) \rightarrow\left(3 p_{3 / 2}\right)$ relevant to $1^{-} \leftrightarrow 2_{1,2}^{+}$is comparable to transition $\left(\right.$cont. $\left.d_{5 / 2}\right) \rightarrow\left(2 f_{7 / 2}\right)$ relevant to $1^{-} \leftrightarrow 0_{\mathrm{g}}^{+}$. In the GDR region $(E \approx 10-17 \mathrm{MeV})$, in contrast, the decay to the ground state is dominant because the lack of the E1 transition strengths to the $2_{1}^{+}$and $2_{2}^{+}$. Note that the E2 decay probability to the $3_{1}^{-}$state is negligibly small $(<0.01 \%)$ and not visible in the scale of Fig, 6 .

\section{C. $2^{+}$states: low-lying states and GQRs}

Here we discuss excited $2^{+}$states with focus on the GQR's and the low-lying $2^{+}$states.

Figure $7(\mathrm{~b})$ shows the strength function $S\left(D_{\mathrm{IV}} ; 3_{1}^{-}, 2^{+} ; E\right)$ for the E1 transitions from $3_{1}^{-}$to the excited $2^{+}$states. A peak around $E \approx 12 \mathrm{MeV}$ corresponds to the transition from the low-lying collective $3_{1}^{-}$state to ISGQR. We also observe another small peak at $E \approx 1 \mathrm{MeV}$. This is the transitions between the low-lying $2_{1,2}^{+}$states and the collective $3_{1}^{-}$ 


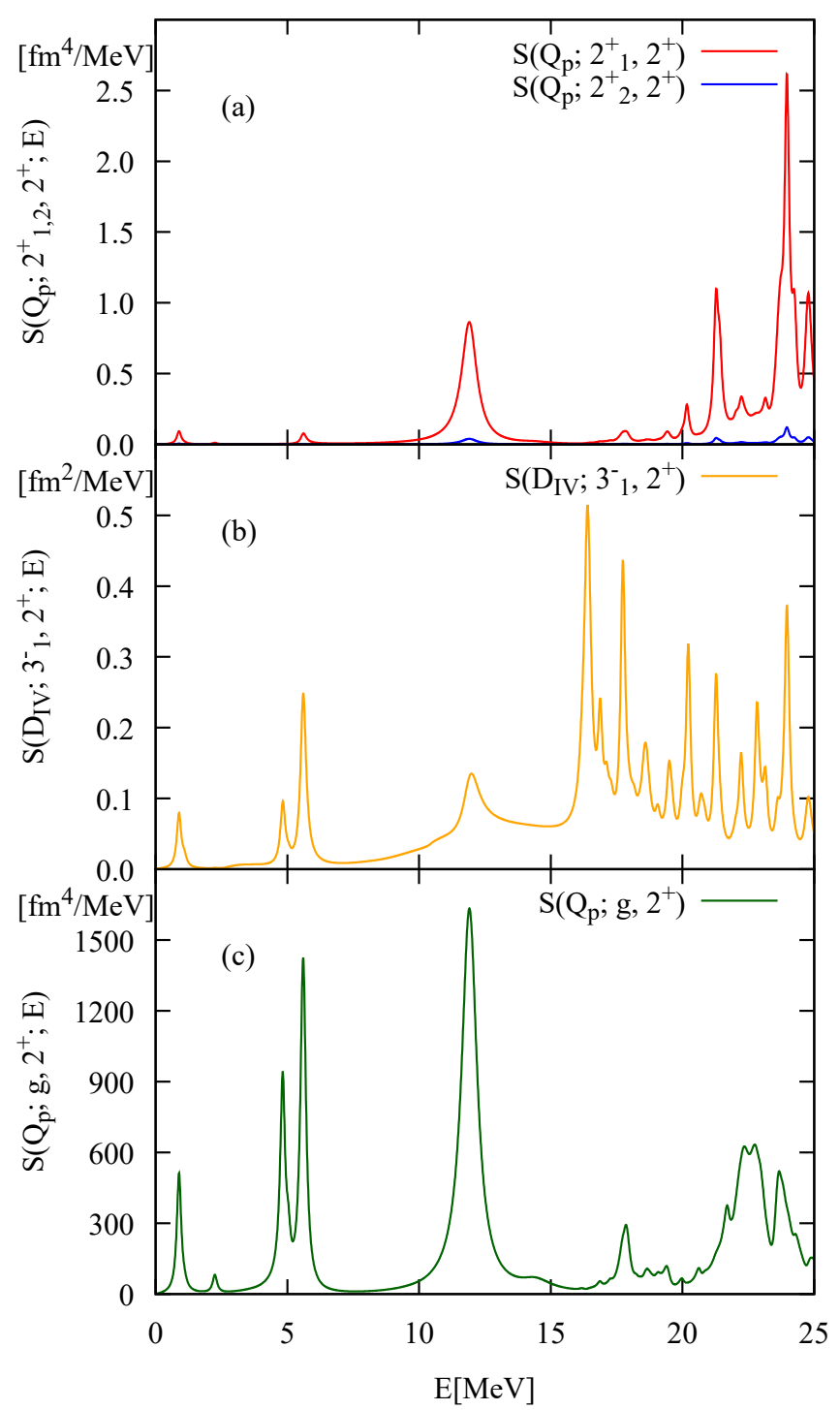

FIG. 7. (a)The E2 strength functions $S\left(Q_{\mathrm{p}} ; 2_{1,2}^{+}, 2^{+} ; E\right)$ for transitions from $2_{1,2}^{+}$to $2^{+}$states. (b) The E1 strength function $S\left(D_{\mathrm{IV}} ; 3_{1}^{-}, 2^{+} ; E\right)$ for transitions from $3_{1}^{-}$to $2^{+}$states. (c) The E2 strength function $S\left(Q_{\mathrm{p}} ; \mathrm{g}, 2^{+} ; E\right)$ for transitions from the ground state to $2^{+}$states. The horizontal axis is the excitation energy of the $2^{+}$states.

state. (Note that the two $2^{+}$states are not resolved due to the smoothing with $\eta=0.1 \mathrm{MeV}$.) These transitions can be described only if the correlation and the collectivity are taken into account in the theory. Several peaks at high energy region $E \gtrsim 16 \mathrm{MeV}$ and $E \sim 5 \mathrm{MeV}$ 


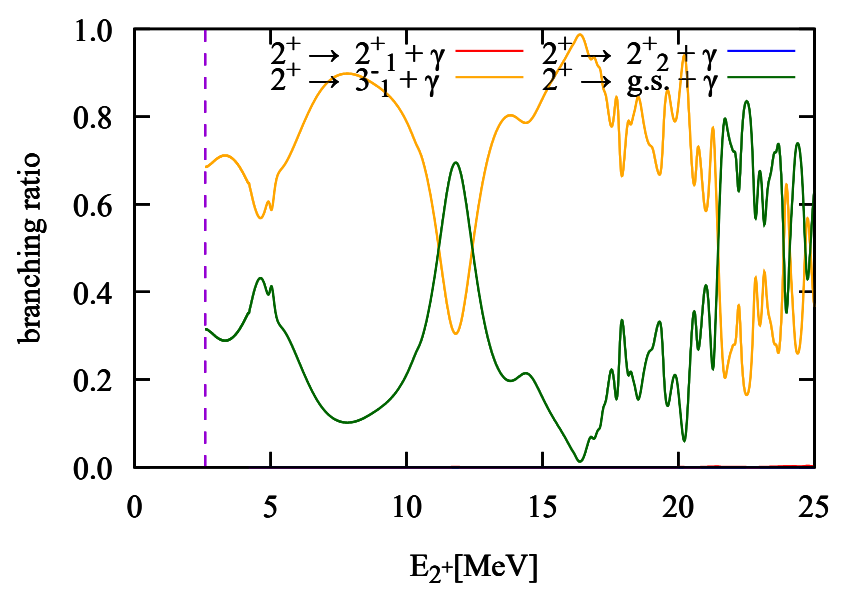

FIG. 8. The branching ratios of photo-emission decays from the excited $2^{+}$states to the low-lying $3_{1}^{-}$state (yellow curve) and the ground state (green one) in ${ }^{140} \mathrm{Sn}$. The strengths associated with the low-lying $2^{+}$states below the neutron separation energy (dotted line) are removed. Horizontal axis is the excitation energy of the $2^{+}$states. The branching ratio to the $2_{1,2}^{+}$states are invisibly small.

correspond to particle-hole configurations of both neutrons and protons, and existence of these transitions can be understood in terms of the same argument as that for the E2 transitions from $3_{1}^{-}$to $1^{-}$states.

The roles of the correlation and the collectivity are also seen in the E2 transitions between $2_{1,2}^{+}$and higher-lying $2^{+}$states, shown in the strength function $S\left(Q_{\mathrm{p}} ; 2_{1,2}^{+}, 2^{+} ; E\right)$ (Fig. 7 (a) ). An example is the transition between $2_{1}^{+}$and ISGQR. This transition strength appears only if configuration mixing of the proton particle-hole components is taken into account in the $2_{1}^{+}$state. Note however that the overall strengths in $S\left(Q_{\mathrm{p}} ; 2_{1,2}^{+}, 2^{+} ; E\right)$ between $2_{1,2}^{+}$and higher-lying $2^{+}$states (panel (a)) are significantly smaller than the E2 transition strengths from the ground state $\left(S\left(Q_{\mathrm{p}} ; \mathrm{g}, 2^{+} ; E\right)\right.$ shown panel $\left.(\mathrm{c})\right)$ due to the small admixture of proton configurations. The $2_{2}^{+}$state has even smaller admixture, as is suggested by the very small $B\left(E 2, g s \rightarrow 2_{2}^{+}\right)$(see the inset of Fig $2(\mathrm{~b})$ ), resulting in much smaller strengths than that of 
$2_{1}^{+}$

Figure 8 shows the branching ratio of the photo-emission decays from the excited $2^{+}$ states to the ground state (E2) and the $3_{1}^{-}$state (E1). The branching ratios of the E2 decays to the $2_{1,2}^{+}$states is not shown here since they are negligibly small. A gross behavior is that the E1 decay probability to $3_{1}^{-}$state is larger than the E2 decays to the ground state in most of the plotted energy range except in the isoscalar and isovector GQR regions ( $E \sim 12 \mathrm{MeV}$ and $E \sim 22-25 \mathrm{MeV}$ ), In these two energy regions, the collectivity of the GQR's enhances the E2 transition probability to the ground state and hence the branching ratio. The collectivity of the ISGDR causes also enhancement the transition to the $3_{1}^{-}$state, but to a smaller extent than that to the ground state.

\section{D. $3^{-}$states: continuum, low-lying and high-lying collective states}

Concerning the excited $3^{-}$states, we observe additional new features as well as similar behaviours to those found in the above examples.

Figure $9\left(\right.$ a) shows the strength function $S\left(D_{\mathrm{IV}} ; 2_{1,2}^{+}, 3^{-} ; E\right)$ for the E1 transition from the low-lying $2_{1,2}^{+}$to the $3^{-}$states. A characteristic feature is continuum strength in the region $2.59\left(=S_{1 n}\right)<E \lesssim 7 \mathrm{MeV}$, as is similarly seen in the strength function for the E1 transition $2_{1,2}^{+} \rightarrow 1^{-}($Fig $3(\mathrm{a}))$. Indeed this feature can be understood in terms of the same argument using two dominant neutron particle-hole configurations $\nu\left[\left(1 h_{9 / 2}\right)\left(2 f_{7 / 2}\right)^{-1}\right]_{2^{+}}$and $\nu\left[\left(3 p_{3 / 2}\right)\left(2 f_{7 / 2}\right)^{-1}\right]_{2^{+}}$in the low-lying $2_{1,2}^{+}$states. Comparing with the unperturbed transitions from these two configurations (Fig,11), we find that the continuum strength is associated with continuum particle-hole states $\nu\left[\left(\text { cont.s } s_{1 / 2}\right)\left(2 f_{7 / 2}\right)^{-1}\right]_{3^{-}}$and $\nu\left[\left(\text { cont. } d_{5 / 2,3 / 2}\right)\left(2 f_{7 / 2}\right)^{-1}\right]_{3^{-}}$, which are excited from the configuration $\nu\left[\left(3 p_{3 / 2}\right)\left(2 f_{7 / 2}\right)^{-1}\right]_{2^{+}}$ by neutron single-particle transition from $3 p_{3 / 2}$ to continuum $s_{1 / 2}$ and $d$ orbits, (cf. the di- 
agram of Fig.10(b)). Other components shown in the diagrams Fig.10(a)(c) and (d) brings three narrow peaks appearing around $E \approx 9-13 \mathrm{MeV}$.

We emphasize that the E1 transition between the low-lying $2_{1,2}^{+}$and the continuum octupole state is different from that between $2_{1,2}^{+}$and the continuum dipole state: The strength of the former rises sharply at the threshold energy $E=2.59 \mathrm{MeV}\left(=S_{1 n}\right)$. This originates from the transition $3 p_{3 / 2} \rightarrow$ cont. $s_{1 / 2}$, where the continuum s-orbit causes a cusp behavior at the threshold. For $1^{-}$, however, the configuration $\nu\left(\right.$ cont. $\left.s_{1 / 2}\right)\left(2 f_{7 / 2}\right)^{-1}$ with the continuum $s$-orbit is forbidden by the angular momentum coupling.

Examples showing the collective effect are remarked also. A small peak at $E \approx 1.8 \mathrm{MeV}$ (below the neutron threshold energy) corresponds to the E1 transition between the low-lying octupole vibrational state $3_{1}^{-}$at $E=1.77 \mathrm{MeV}$ and the low-lying $2_{1,2}^{+}$states. Existence of the low-lying collective state is a peculiar aspect of the $3^{-}$channel.

Another example of the collective effect is seen in Fig. 9(b), which shows the strength function $S\left(Q_{\mathrm{p}} ; 3_{1}^{-}, 3^{-} ; E\right)$ of the E2 transitions between $3_{1}^{-}$and all the RPA excited states with $3^{-}$. A peak at $E=1.77 \mathrm{MeV}$, which corresponds to the diagonal matrix element $\left\langle 3_{1}^{-}|| \mathrm{E} 2|| 3_{1}^{-}\right\rangle$for the low-lying collective state, is significantly enhanced in comparison with the E2 matrix elements associated with other non-collective $3^{-}$states. The enhancement is caused by the collective and surface vibrational character of the $3_{1}^{-}$state.

Figure 12 shows the branching ratio of the photo-emission decays from $3^{-}$states to the ground state (E3 transition), the $2_{1,2}^{+}$states (E1) and the $3_{1}^{-}$state (E2). The E1 transitions feeding to the low-lying $2_{1,2}^{+}$states dominate over the E3 transition to the ground states in all the energy range from the continuum octupole states to the highest energy region $E \sim 20-25 \mathrm{MeV}$. Looking at more details, the branching ratios to the ground state, $2_{1}^{+}, 2_{2}^{+}$, and $3_{1}^{-}$states reflect various structures of the initial $3^{-}$states. For example, the branching 


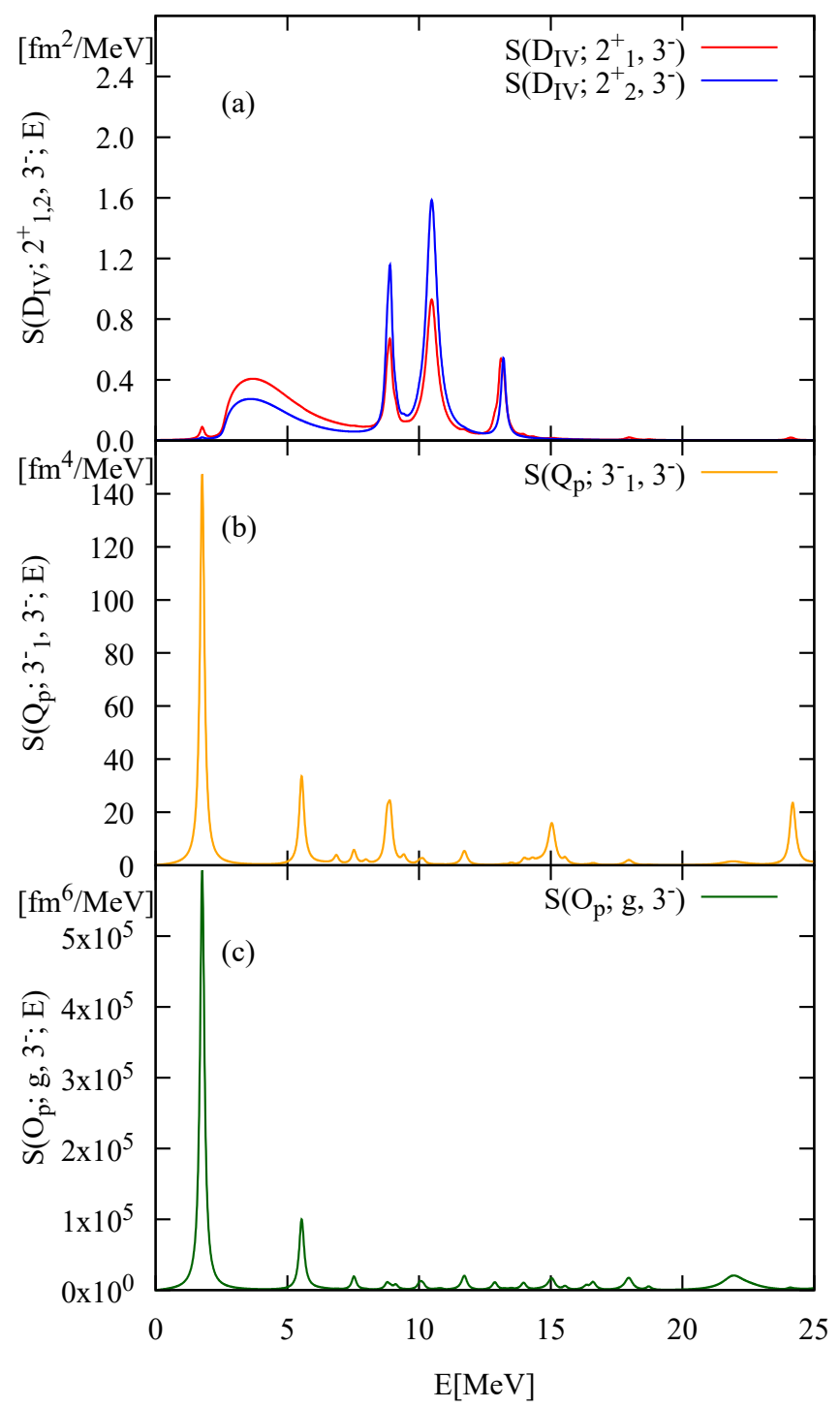

FIG. 9. (a)The E1 strength functions $S\left(D_{\mathrm{IV}} ; 2_{1,2}^{+}, 3^{-} ; E\right)$ for transitions from $2_{1,2}^{+}$to $3^{-}$states. (b) The E2 strength function $S\left(Q_{\mathrm{p}} ; 3_{1}^{-}, 3^{-} ; E\right)$ for transitions from $3_{1}^{-}$to $3^{-}$states. (c) The E3 strength function $S\left(O_{\mathrm{p}} ; \mathrm{g}, 3^{-} ; E\right)$ for transitions from the ground state to $3^{-}$states. The horizontal axis is the excitation energy of the $3^{-}$states.

ratios for decays from continuum $3^{-}$state around $2.59<E \lesssim 7 \mathrm{MeV}$ to the two lowlying $2_{1}^{+}$and $2_{2}^{+}$states are well accounted for by the mixing amplitudes of the configuration $\nu\left[\left(2 p_{3 / 2}\right)\left(2 f_{7 / 2}\right)^{-1}\right]_{2^{+}}$in $2_{1}^{+}$and $2_{2}^{+}$(cf. Table II). This indicates that the continuum octupole 


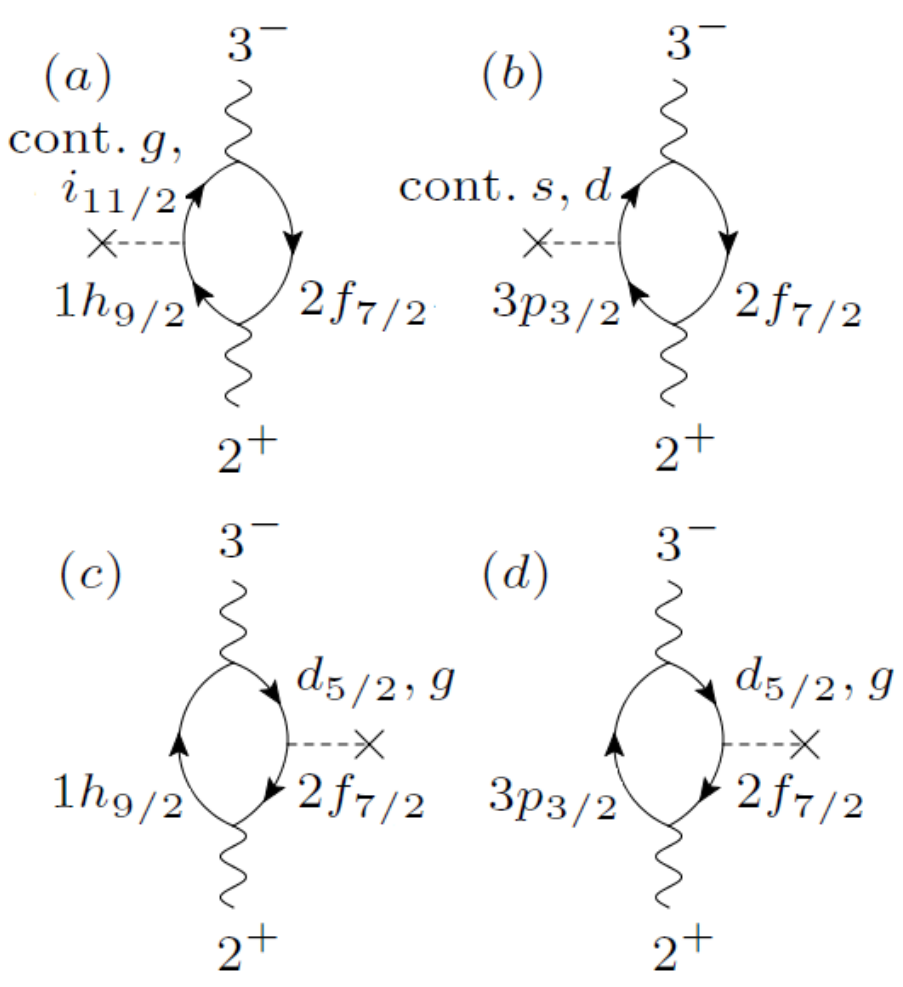

FIG. 10. The diagrams representing dominant components of transition between the exited $3^{-}$ states and the low-lying $2_{1,2}^{+}$in ${ }^{140} \mathrm{Sn}$.

states in this energy region is uncorrelated particle-hole excitations $\nu\left[\left(\text { cont. } s_{1 / 2}\right)\left(2 f_{7 / 2}\right)^{-1}\right]_{3^{-}}$ and $\nu\left[\left(\text { cont. } d_{3 / 2}, 1 / 2\right)\left(2 f_{7 / 2}\right)^{-1}\right]_{3^{-}}$. The branching ratio for transitions from the vibrational collective $3_{1}^{-}$state to $2_{1}^{+}$and $2_{2}^{+}$(the crosses at $E=1.77 \mathrm{MeV}$ ) are different from that of the continuum states, reflecting significant configuration mixing in the $3_{1}^{-}$state (Table III). Around $E \sim 22 \mathrm{MeV}$, collectivity of the high-lying octupole vibrational state enhances the branching to the ground state.

\section{CONCLUSION}

The continuum random phase approximation (cRPA), referred also to the linear response theory, describes both collective and non-collective particle-hole excitations as well as their 


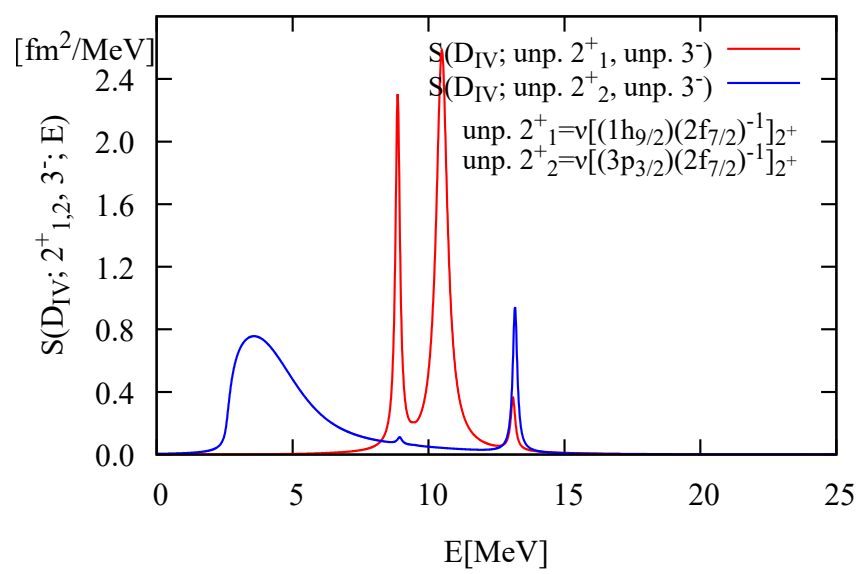

FIG. 11. The E1 strength functions $S\left(D_{\mathrm{IV}} ; \nu\left[\left(1 h_{9 / 2}\right)\left(2 f_{7 / 2}\right)^{-1}\right]_{2^{+}}, 3^{-} ; E\right)$ and $S\left(D_{\mathrm{IV}} ; \nu\left[\left(3 p_{3 / 2}\right)\left(2 f_{7 / 2}\right)^{-1}\right]_{2^{+}}, 3^{-} ; E\right) \quad$ for $\quad$ transitions from the neutron $1 \mathrm{p}-1 \mathrm{~h}$ states $\nu\left[\left(1 h_{9 / 2}\right)\left(2 f_{7 / 2}\right)^{-1}\right]_{2^{+}}$and $\nu\left[\left(3 p_{3 / 2}\right)\left(2 f_{7 / 2}\right)^{-1}\right]_{2^{+}}$to unperturbed $3^{-}$states. The horizontal axis is the excitation energy of the $3^{-}$states.

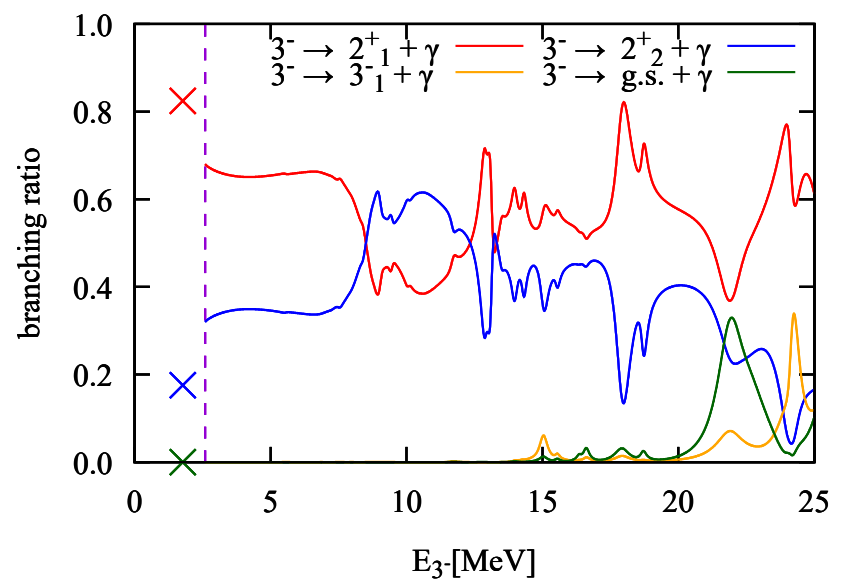

FIG. 12. The branching ratios of photo-emission decays from the excited $3^{-}$states to the ground state (green curve), the $2_{1}^{+}$state (red curve), the $2_{2}^{+}$state (blue curve) and the $3_{1}^{-}$state (yellow) in ${ }^{140} \mathrm{Sn}$. Horizontal axis is the excitation energy of the $3^{-}$states. The neutron separation energy is $2.59 \mathrm{MeV}$ (dotted line). 
coupling to unbound single-particle orbits which plays key roles in exotic nuclei close to the proton and neutron drip-lines. On the basis of the nuclear density functional theory or the self-consistent Hartree-Fock model, cRPA provides a well-defined scheme to calculate the response function for one-body field, e.g. the electromagnetic matrix elements, for transitions from the ground state to the excited states. In the present study, we have extended the linear response theory so that one can calculate transitions from a low-lying excited state to the RPA excited states under consideration. This extension enables us to calculate gammadecays from the RPA excited states to a set of the low-lying excited states, and hence the branching ratio and the total decay probability including different final states.

In order to demonstrate the applicability of the extended cRPA we have described the $1^{-}, 2^{+}$and $3^{-}$excitations in a neutron-rich nucleus ${ }^{140} \mathrm{Sn}$. Specifically we discuss the E1, E2 and E3 transitions among low-lying vibrational states, higher-lying giant resonances and the unbound particle-hole states with continuum spectra. An important conclusion is that there exist cases where the branching ratios to the low-lying excited states are larger than or comparable with that to the ground state. Furthermore we have demonstrated that the extended cRPA enables us to analyze microscopic mechanisms how the transitions between the excited states reflect the nature of the correlations in both the initial and final states.

Let us remark future developments of the present study. Firstly, we plan to describe the radiative neutron-capture reaction of neutron-rich nuclei by applying the extended cRPA. In a preceding work [12] we have formulated the theory of the direct neutron-capture reaction in which the reaction proceeds via the RPA correlated states, but we described only the limited process where the final state of the gamma-decays from the RPA states is the ground state of the synthesized nucleus. Applying the extended cRPA, we can include decay channels populating the low-lying excited states, and hence provides more realistic description of the 
radiative neutron-capture reactions. Secondly, we plan to take into account the pair correlation which need to be included when the theory is applied to open-shell nuclei. Following Ref. [23], this can be achieved by replacing the RPA with the quasiparticle random phase approximation (QRPA).

\section{ACKNOWLEDGMENTS}

The authors thank Kazuyuki Sekizawa for valuable discussions. This work was supported by the JSPS KAKENHI (Grant No. 20K03945).

\section{Appendix A: Pseudo transition density-matrix in the linear response formalism}

The pseudo transition density-matrix can be calculated as follows. Suppose that we describe the low-lying excited state $|i\rangle$ using the linear response equation

$\delta \rho^{\prime}\left(\mathbf{r}_{x}, \omega\right)=\sum_{\sigma_{x}} \int d x^{\prime} R_{0}\left(x, x ; x^{\prime}, x^{\prime} ; \omega\right) \frac{\delta U}{\delta \rho}\left(\mathbf{r}_{x^{\prime}}\right) \delta \rho^{\prime}\left(\mathbf{r}_{x^{\prime}}, \omega\right)+\sum_{\sigma_{x}} \int d x^{\prime} R_{0}\left(x, x ; x^{\prime}, x^{\prime} ; \omega\right) f^{\prime}\left(\mathbf{r}_{x^{\prime}}\right)$

and an external perturbation $\hat{M}^{\prime}=\int d x f^{\prime}\left(\mathbf{r}_{x}\right) \hat{\rho}(x)$, which is suitable to excite $|i\rangle$. (Equation (A1) is essentially the same as Eq. 17) except the difference in the external perturbation. We put prime ' to the density response $\delta \rho^{\prime}\left(\mathbf{r}_{x}, \omega\right)$ in order to distinguish it from $\delta \rho\left(\mathbf{r}_{x}, \omega\right)$ in Eq.(17).) We can also consider the extended linear response equation for the density-matrix response $\delta \rho^{\prime}(x, y, \omega)$ in terms of an equation similar to Eq. 16.

The low-lying RPA state $|i\rangle$ appears as a pole at $\omega_{i}=\left(E_{i}-E_{0}\right) / \hbar$, in $\delta \rho^{\prime}\left(\mathbf{r}_{x}, \omega\right)$ provided that $|i\rangle$ is a discrete bound state. The transition density $\rho_{i}^{(\operatorname{tr})}\left(\mathbf{r}_{x}, \omega\right) \equiv \sum_{\sigma_{x}}\langle 0|\hat{\rho}(x)| i\rangle$ 
corresponds to the residue of $\delta \rho^{\prime}\left(\mathbf{r}_{x}, \omega\right)$ at the pole, and thus can be calculated with

$$
\rho_{i}^{(\operatorname{tr})}\left(\mathbf{r}_{x}, \omega\right)=C^{\prime} \operatorname{Im} \delta \rho^{\prime}\left(\mathbf{r}_{x}, \omega_{i}\right)
$$

where $C^{\prime}$ is a normalization constant. Similarly the transition density-matrix $\rho_{i}^{(\operatorname{tr})}(x, y)$ is also given by

$$
\begin{aligned}
\rho_{i}^{(\operatorname{tr})}(x, y) & =C^{\prime} \operatorname{Im} \delta \rho^{\prime}\left(x, y, \omega_{i}\right) \\
& =C^{\prime} \operatorname{Im}\left\{\int d x^{\prime} R_{0}\left(x, y ; x^{\prime}, x^{\prime} ; \omega_{i}\right) \frac{\delta U}{\delta \rho}\left(\mathbf{r}_{x^{\prime}}\right) \delta \rho^{\prime}\left(\mathbf{r}_{x}, \omega_{i}\right)+\int d x^{\prime} R_{0}\left(x, y ; x^{\prime}, x^{\prime} ; \omega_{i}\right) f^{\prime}\left(\mathbf{r}_{x^{\prime}}\right)\right\}
\end{aligned}
$$

As we discussed for Eq. 11), the pseudo transition density-matrix $\bar{\rho}_{i}^{(\operatorname{tr})}(x, y)$ has the same structure as that of the transition density-matrix except the sign of the backward amplitudes. Thus it is calculated with

$\bar{\rho}_{i}^{(\operatorname{tr})}(x, y)=C^{\prime} \operatorname{Im}\left\{\int d x^{\prime} \bar{R}_{0}\left(x, y ; x^{\prime}, x^{\prime} ; \omega_{i}\right) \frac{\delta U}{\delta \rho}\left(\mathbf{r}_{x^{\prime}}\right) \delta \rho^{\prime}\left(\mathbf{r}_{x}, \omega_{i}\right)+\int d x^{\prime} \bar{R}_{0}\left(x, y ; x^{\prime}, x^{\prime} ; \omega_{i}\right) f^{\prime}\left(\mathbf{r}_{x^{\prime}}\right)\right\}$,

$$
\begin{array}{r}
\bar{R}_{0}\left(x, y ; y^{\prime}, x^{\prime} ; \omega\right) \equiv \sum_{h}\left\{\phi_{h}^{*}(y) \bar{G}_{0}\left(x, x^{\prime}, \epsilon_{h}+\hbar \omega+i \eta\right) \phi_{h}\left(y^{\prime}\right)\right. \\
\left.-\phi_{h}^{*}\left(x^{\prime}\right) \bar{G}_{0}\left(y^{\prime}, y, \epsilon_{h}-\hbar \omega-i \eta\right) \phi_{h}(x)\right\}
\end{array}
$$

where the function $\bar{R}_{0}$ is a variant of the unperturbed response function $R_{0}$ with the sign of the second term opposite to that of Eq.(15). Note that the Green's function $G_{0}$ is replaced with

$$
\bar{G}_{0}\left(x, x^{\prime}, e\right) \equiv G_{0}\left(x, x^{\prime}, e\right)-\sum_{h} \frac{\phi_{h}(x) \phi_{h}^{*}\left(x^{\prime}\right)}{e-\epsilon_{h}} .
$$

so that the contribution of the hole orbits in the Green's function are removed. This replacement is necessary to remove hole-hole components in $\bar{R}_{0}$, which are automatically canceled out in the original unperturbed response function $R_{0}$. 


\section{Appendix B: Response functions for spherical mean-field}

Assuming the spherical symmetry of the mean-field, we represent the single-particle wave function by $\phi_{n l j m}(x)=Y_{l j m}(\hat{x}) \frac{1}{r_{x}} \phi_{n l j}\left(r_{x}\right)$, where $r_{x}$ and $\hat{x} \equiv\left(\hat{\mathbf{r}}_{x}, \sigma_{x}\right)$ are the radial and anglespin variables, respectively and $Y_{l j m}(\hat{x})$ is the spin spherical harmonics with the angular quantum numbers $\operatorname{ljm}$.

The single-particle Green's function is given by

$$
G_{0}\left(x, x^{\prime}, E\right)=\sum_{l j m} Y_{l j m}(\hat{x}) \frac{1}{r_{x} r_{x^{\prime}}} G_{0, l j}\left(r_{x}, r_{x^{\prime}}, E\right) Y_{l j m}^{*}\left(\hat{x}^{\prime}\right)
$$

whose radial part can be constructed exactly as

$G_{0, l j}\left(r_{x}, r_{x}^{\prime}, E\right)=\frac{2 m}{\hbar^{2}} \frac{1}{W\left(\phi_{1, l j}, \phi_{2, l j}\right)}\left\{\phi_{1, l j}\left(r_{x}^{\prime}\right) \phi_{2, l j}\left(r_{x}\right) \theta\left(r_{x}-r_{x}^{\prime}\right)+\phi_{1, l j}\left(r_{x}\right) \phi_{2, l j}\left(r_{x}^{\prime}\right) \theta\left(r_{x}^{\prime}-r_{x}\right)\right\}$

in terms of the regular radial wave $\phi_{1, l j}(r)$ and the outgoing wave $\phi_{2, l j}(r)$ with a given complex energy $E$. $W$ is the Wronskian.

The unperturbed response function for density matrix and non-local one-body operators is represented by

$R_{0}\left(x, y ; y^{\prime}, x^{\prime} ; \omega\right)=\sum_{l j m, l^{\prime} j^{\prime} m^{\prime}} Y_{l^{\prime} j^{\prime} m^{\prime}}(\hat{x}) Y_{l j m}^{*}(\hat{y}) \frac{1}{r_{x} r_{y} r_{y^{\prime}} r_{x^{\prime}}} R_{0, l^{\prime} j^{\prime}, l j}\left(r_{x}, r_{y} ; r_{y^{\prime}}, r_{x^{\prime}} ; \omega\right) Y_{l j m}\left(\hat{y}^{\prime}\right) Y_{l^{\prime} j^{\prime} m^{\prime}}^{*}\left(\hat{x}^{\prime}\right)$.

Here the radial unperturbed response function is given by

$$
\begin{aligned}
R_{0, l^{\prime} j^{\prime}, l j}\left(r_{x}, r_{y} ; r_{y^{\prime}}, r_{x^{\prime}} ; \omega\right)=\sum_{n} & \left\{\phi_{n l j}^{*}\left(r_{y}\right) G_{0, l^{\prime} j^{\prime}}\left(r_{x}, r_{x^{\prime}}, \epsilon_{n l j}+\hbar \omega+i \eta\right) \phi_{n l j}\left(r_{y^{\prime}}\right) \theta\left(\epsilon_{F}-\epsilon_{n l j}\right)\right. \\
& \left.+\phi_{n l^{\prime} j^{\prime}}^{*}\left(r_{x^{\prime}}\right) G_{0, l j}\left(r_{y^{\prime}}, r_{y}, \epsilon_{n l^{\prime} j^{\prime}}-\hbar \omega-i \eta\right) \phi_{n l^{\prime} j^{\prime}}\left(r_{x}\right) \theta\left(\epsilon_{F}-\epsilon_{n l^{\prime} j^{\prime}}\right)\right\} .
\end{aligned}
$$


where $\phi_{n l j}(r)$ is a radial wave function of the single-particle states occupied in the ground state.

We define the creation operator of an RPA excited state and its forward and backward amplitudes by

$$
\begin{aligned}
\hat{O}_{i L_{i} M_{i}}^{\dagger} & =\sum_{p h}\left\{X_{p h}^{i}\left[a_{p}^{\dagger} a_{h}\right]_{L_{i} M_{i}}-Y_{p h}^{i}\left[a_{h}^{\dagger} a_{p}\right]_{L_{i} M_{i}}\right\} \\
{\left[a_{p}^{\dagger} a_{h}\right]_{L_{i} M_{i}} } & =\sum_{m_{p} m_{h}}\left\langle j_{p} m_{p} j_{h} m_{h} \mid L_{i} M_{i}\right\rangle a_{n_{p} l_{p} j_{p} m_{p}}^{\dagger} a_{n_{h} \widetilde{l_{h} j_{h} m_{h}}},
\end{aligned}
$$

where $a_{\text {nljm }}$ is the time reversal of the Fermion annihilation operator $a_{n l j m}$.

[1] P. G. Hansen and B. Jonson, Europhys. Lett. 4, 409 (1987).

[2] Y. Suzuki, K. Ikeda, and H. Sato, Prog. Theor. Phys. 83, 180 (1990).

[3] G. F. Bertsch and H. Esbensen, Ann. Phys. A 209, 327 (1991).

[4] N. Paar, D. Vretenar, E. Khan, and G. Colò, Rep. Prog. Phys. 70, 691 (2007).

[5] D. Savran, T. Aumann, and A. Zilges, Prog. in Part. and Nucl. Phys. 70, 210 (2013).

[6] T. Aumann, Eur. Phys. J. A 55, 234 (2019).

[7] T. Aumann and T. Nakamura, Phys. Scr. T152, 014012 (2013).

[8] S. Goriely, Phys. Lett. B 436, 10 (1998).

[9] M. Arnould, S. Goriely, and K. Takahashi, Phys. Rep. 450, 97 (2007).

[10] G. J. Mathews, A. Mengoni, F.-K. Thielemann, and W. A. Fowler, Astrophys. J. 270, 740 (1983).

[11] S. Goriely, Astron. Astrophys. 325, 414 (1997).

[12] M. Matsuo, Phys. Rev. C 91, 034604 (2015).

[13] A. M. Lane and J. E. Lynn, Nucl. Phys. 17, 563 (1960). 
[14] S. Raman, R. F. Carlton, J. C. Wells, E. T. Jurney, and J. E. Lynn, Phys. Rev. C 32, 18 (1985).

[15] A. Mengoni, T. Otsuka, and M. Ishihara, Phys. Rev. C 52, R2334 (1995).

[16] T. Rauscher, R. Bieber, H.Oberhummer, K.-L. Kratz, J. Dobaczewski, P. Möller, and M. M. Sharma, Phys. Rev. C 57, 2031 (1998).

[17] T. Rauscher, Nucl. Phys. A 834, 635c (2010).

[18] Y. Xu and S. Goriely, Phys. Rev. C 86, 045801 (2012).

[19] L. Bonneau, T. Kawano, T. Watanabe, and S.Chiba, Phys. Rev. C 75, 054618 (2007).

[20] S. Chiba, H. Koura, T. Hayakawa, T. Maruyama, T. Kawano, and T. Kajino, Phys. Rev. C 77, 015809 (2008).

[21] G. F. Bertsch and S. F. Tsai, Phys. Rep. 18, 125 (1975).

[22] S. Shlomo and G. F. Bertsch, Nucl. Phys. A 243, 507 (1975).

[23] M. Matsuo, Nucl. Phys. A 696, 371 (2001).

[24] A. Zangwill and P. Soven, Phys. Rev. A 21, 1561 (1980).

[25] P. Ring and P. Schuck, The Nuclear Many-Body problem (Springer-Verlag, Berlin, 1980).

[26] Mass explorer, http://massexplorer.frib.msu.edu/content/DFTMassTables.html.

[27] H. Shimoyama and M. Matsuo, Phys. Rev. C 88, 054308 (2013). 